\title{
1 Genotypic similarity among algal symbionts corresponds to associations with closely
}

2 related coral hosts

3

4 Hannah G. Reich ${ }^{1,2^{*}}$, Sheila A. Kitchen ${ }^{1,3^{*}}$, Kathryn H. Stankiewicz ${ }^{1}$, Meghann Devlin-Durante ${ }^{1}$,

$5 \quad$ Nicole D. Fogarty ${ }^{4}$, Iliana B. Baums ${ }^{1 *}$

6

$7 \quad{ }^{1}$ Department of Biology, The Pennsylvania State University, University Park, PA 16801 USA

$8 \quad{ }^{2}$ Present address: Department of Biological Sciences, University of Rhode Island, Kingston, RI

9 02881, USA

$10{ }^{3}$ Present address: Division of Biology and Biological Engineering, California Institute of

11 Technology, Pasadena, CA 91125

$12{ }^{4}$ Department of Biology and Marine Biology, Center for Marine Science, University of North

13 Carolina Wilmington, Wilmington, NC 28409

15 Correspondence: hgreigh16@gmail.com; sak3097@caltech.edu; baums@psu.edu

16 Keywords: Acropora, hybrid, niche diversification, single-nucleotide polymorphisms,

17 Symbiodiniaceae, symbiosis

18 Running title: Allelic variation of an endangered coral symbiont

Abstract

21 Mutualisms where hosts are coupled metabolically to their symbionts often exhibit high partner

22 fidelity. Most reef-building corals form obligate symbioses with specific species of

23 photosymbionts, dinoflagellates in the family Symbiodiniaceae, despite needing to acquire 
24 symbionts early in their development from environmental sources. Three Caribbean acroporids

25 (Acropora palmata, A. cervicornis, and their hybrid A. prolifera) are geographically sympatric

26 across much of their range in the greater Caribbean, but often occupy different depth and light

27 habitats. Both species and their hybrid associate with Symbiodinium 'fitti', a genetically diverse

28 species of symbiont that is specific to these hosts. Since the physiology of the dinoflagellate

29 partner is strongly influenced by light (and therefore depth), we investigated whether S. 'fitti'

30 populations from each host source were differentiated genetically. We generated shallow genome

31 sequences of acroporid colonies sampled from across the Caribbean. Single Nucleotide

32 Polymorphisms (SNPs) among $S$. 'fitti' strains were identified by aligning sequences to a $\sim 600$

$33 \mathrm{Mb}$ draft assembly of the $S$. 'fitti' genome, assembled from an A. cervicornis metagenome.

34 Phylogenomic and multivariate analyses revealed that allelic variation among $S$. 'fitti' partitioned

35 to each host species, as well as their hybrid, rather than by biogeographic origin. This is

36 particularly noteworthy because the hybrid, $A$. prolifera, has a sparse fossil record and may be of

37 relatively recent origin. Many of the SNPs putatively under selection were non-synonymous

38 mutations predicted to alter protein efficiency. Differences in allele frequency among S. 'fitti'

39 populations from each host taxon may correspond to distinct phenotypes that thrive in the

40 different cellular environments found in each acroporid. The non-random sorting among

41 genetically diverse strains, or genotypes, to different hosts could be the basis for lineage

42 diversification via disruptive selection, leading to ecological specialization and ultimately

43 speciation. 
Ecosystem services provided by coral-dinoflagellate mutualisms rival the contributions of

47 other widely studied symbioses (ex: tubeworms and bacteria, yeast and termites, plant and

48 fungi). In the coral-dinoflagellate mutualism, each partner benefits as the coral receives

49 photosynthetic sugars from their dinoflagellate symbionts and the algal symbiont receives

50 nutrients and protection in return (Trench 1979). Though decades of investigations have probed

51 the causes and consequences of coral-algal dysbiosis (ex: coral bleaching), we are still gathering

52 information on their basic biology (Cziesielski, Schmidt-Roach, \& Aranda 2019). Specifically,

53 little is known about the intraspecies level of co-evolutionary dynamics between host and their

54 symbionts. The interdependence of the partners adds complexity to the system as each partner is

55 selected in the context of the other.

56 Understanding the mechanisms that promote the apparent fidelity of reef-building corals

57 towards one endosymbiotic dinoflagellate species (Symbiodiniaceae), despite having the

58 opportunity to horizontally acquire symbionts, is important in light of rapid climate change. This

59 is because the ability of corals to cope with heat stress by "shuffling" their endosymbiont

60 communities to a more heat tolerant lineage (Little, Oppen, \& Willis 2004; Silverstein, Cunning,

$61 \&$ Baker 2017) may be limited. Corals have co-evolved with the Symbiodiniaceae since the

62 Jurassic period (LaJeunesse et al. 2018) and, over time, may have become uniquely adapted to

63 their symbionts (Goulet 2006; LaJeunesse et al. 2018; LaJeunesse et al. 2004; Lewis, Chan, \&

64 LaJeunesse 2019; Parkinson, Coffroth, \& LaJeunesse 2015b; Thornhill, Fitt, \& Schmidt 2006a).

65 While juvenile corals often host several symbiont species, this community wanes over time to

66 the dominant symbiont species (Abrego, Van Oppen, \& Willis 2009; Coffroth, Goulet, \& Santos

67 2001; Poland \& Coffroth 2017). This suggests that corals are most compatible with their

68 dominant symbiont species and foreign pairings might be maladaptive, at least under current 
conditions (Cunning, Gillette, Capo, Galvez, \& Baker 2015; Pettay, Wham, Smith, IglesiasPrieto, \& LaJeunesse 2015).

A central question in coral reef science is whether coral-dinoflagellate symbioses can adapt to increasing sea surface temperatures over ecological time scales, especially if shuffling of symbiont partners is restricted (Goulet 2006). Genetic variation present within algal species has remained largely unstudied as a source that may fuel such adaptation (Buerger et al. 2020; Parkinson, Banaszak, Altman, LaJeunesse, \& Baums 2015a). The potential for genetic variation within algal species to fuel adaptation to changing conditions can be assessed in the laboratory via experimental evolution experiments where algal strains are selected over several generations under heat stress conditions (Baker et al. 2018; Buerger et al. 2020; Chakravarti \& van Oppen 2018). In one instance, Symbiodiniaceae strains adapted to heat stress selection in vitro but once introduced into the coral partner, gains were not always retained highlighting the complexity of adaptation in the context of mutualistic partners (Buerger et al. 2020).

Alternatively, field studies may assess the long-term influence of selective factors such as a strong light gradient on the genetic variation of Symbiodiniaceae by taking advantage of depth stratification found in their coral hosts (Bongaerts et al. 2015a; Serrano et al. 2016). While sharing a geographic range, Caribbean Acropora species often differentiate across a depth and light gradient; $A$. cervicornis occupies a lower light habitat ( $\sim 10 \mathrm{~m}$ depth) relative to its highlight dwelling ( $\sim 3 \mathrm{~m}$ depth) sibling species $A$. palmata and their hybrid A. prolifera $(\sim 1 \mathrm{~m}$ depth; (Fogarty 2012; Goreau 1959; LaJeunesse 2002). All three taxa harbor the dinoflagellate endosymbiont Symbiodinium 'fitti' (ITS2 type A3), which is distinct from other Symbiodinium A3 lineages found in giant clams and other cnidarians (Kemp et al. 2015; Lee et al. 2015; Pinzón et al. 2015; Shoguchi et al. 2018). The variation of morphology between the three taxa ranges 
92 from broad, moose antler branches to thinner, stag antler branches results in differences in the

93 flow and light field within and around the colonies (Enríquez, Méndez, Hoegh-Guldberg, \&

94 Iglesias-Prieto 2017; Gladfelter 1983; Gladfelter 2007). Therefore, the persistence of S. 'fitti' in

95 three host taxa at a range of depths across a large geographic region provides a unique

96 opportunity to study how evolutionary history, geography, natural ecology, and biophysical

97 parameters may contribute to adaptation and co-evolution in the coral holobiont.

99 influenced by differences in their reproduction and dispersal strategies (Reviewed in Thornhill,

100 Howells, Wham, Steury, \& Santos 2017). Caribbean acroporid corals reproduce via production

101 of meiotic, planktonic larvae and also disperse locally via fragmentation. Gene flow is restricted

102 between eastern and western Caribbean region for A. palmata and A. cervicornis (Baums, Miller,

$103 \&$ Hellberg 2005, 2006). Within each region, further population structure is observed but the

104 specifics differ between species with A. cervicornis showing generally more fine-scale

105 differentiation then A. palmata (Baums et al. 2005, 2006; Devlin-Durante \& Baums 2017;

106 Hemond \& Vollmer 2010; Kitchen et al. 2019; Vollmer \& Palumbi 2002, 2006). A. palmata and

107 A. cervicornis have been present in the fossil record since the late Pliocene ( 2.6-3.6 Mya)

108 whereas the hybrid A. prolifera is mostly absent from the fossil record (Budd \& Johnson 1999;

109 McNeill, Budd, \& Borne 1997; Precht, Vollmer, Modys, \& Kaufman 2019). Although $A$.

110 prolifera produces viable eggs and sperm, molecular analyses indicates that F2 adults are very

111 rare or absent while backcrosses with either parent species occur occassionally (Kitchen et al.

112 2020; Van Oppen, Willis, Vugt, \& Miller 2000; Vollmer \& Palumbi 2002).

113 The population structure and genotypic diversity of S. 'fitti' has received less attention

114 and at a coarser level of genomic resolution (Baums et al. 2019; Baums, Devlin-Durante, \& 
115 LaJeunesse 2014; Thornhill et al. 2017). Despite that, higher levels of population genetic structure are documented in S. 'fitti' when compared to one of its hosts, A. palmata (Baums et al.

117 2014). S. 'fitti' cells divide mitotically within the host and water column dispersal appears

118 limited (Fitt \& Trench 1983; Thornhill et al. 2017). Accordingly, the majority of Caribbean

119 acroporids colonies host a single strain of $S$. 'fitti' and maintain long-term fidelity to that strain

120 (Baums et al. 2014; O’Donnell, Lohr, Bartels, Baums, \& Patterson 2018). However, sexual

121 reproduction in Symbiodiniaceae has not been completely ruled out as a reproductive strategy

122 because meiotic machinery has been detected in genomic data (Bellantuono, Dougan, Granados-

123 Cifuentes, \& Rodriguez-Lanetty 2019; Chi, Parrow, \& Dunthorn 2014; Levin et al. 2016; Shah,

124 Chen, Bhattacharya, \& Chan 2020) and recombination is evident in population genetic data

125 (Baums et al. 2014). These contrasting life-history strategies may contribute to the higher levels

126 of population structure of $S$. 'fitti' compared to their acroporid hosts throughout the Caribbean

127 (Baums et al. 2014; Thornhill et al. 2017).

128 Here, we describe fine-scale genetic differences in S. 'fitti' strains across its three host

129 taxa spanning the geographic distribution of the mutualism. A draft $S$. 'fitti' genome assembly

130 was constructed from $A$. cervicornis metagenomic sequences and compared to other

131 Symbiodiniaceae genomic resources. Variation in genome-wide single nucleotide

132 polymorphisms (SNPs) were investigated in S. 'fitti' and scanned for mutations that may change

133 protein structure and function. Lastly, the potential biological and evolutionary ramifications of

134 the allelic composition of $S$. 'fitti' are discussed.

136 Methods

137 Sample Collection, Sequencing, and Assembly 
Tissue was collected for genome sequencing from 76 acroporids spanning the geographic distribution of $S$. 'fitti' (Fig. 1, Table S1; Kitchen et al. 2019). High molecular weight DNA was

140 isolated from each coral tissue sample using the Qiagen DNeasy kit (Qiagen, Valencia, CA)

141 without prior enrichment for $S$. 'fitti'. Of these samples, one specimen for each species from

142 Florida (A. cervicornis CFL14120 and A. palmata PFL1012) was ‘deeply’ sequenced ( $150 \mathrm{x}$

143 coverage; Kitchen et al. 2019). Paired-end short insert (550 nt) sequencing libraries of the two

144 deeply sequenced samples were constructed with 1.8-2 $\mu$ g sample DNA and the TruSeq DNA

145 PCR-Free kit (Illumina, San Diego, CA). The remaining 74 paired-end short insert (350 nt)

146 sequencing libraries were constructed using $100 \mathrm{ng}$ sample DNA and the TruSeq DNA Nano kit

147 (Illumina, San Diego, CA) with coverage between 8-40x (Kitchen et al. 2019). Deep- and

148 shallow-sequence libraries were pooled separately and sequenced on either Illumina HiSeq 2500 149 or HiSeq 4000 (Table S1, Illumina, San Diego, CA).

Sequencing adaptors and low-quality base calls (Phred score $<25)$ from the 3 ' end of the

151 deeply sequenced $A$. cervicornis metagenome reads were trimmed using cutadapt v 1.6 (Martin

152 2011). After initial filtering, processed reads shorter than 50 bp were discarded and PCR

153 duplicates removed using FastUniq v. 1.1 (Xu et al. 2012). A series of filtering steps were

154 completed to identify the fraction of reads originating from A. cervicornis and Symbiodinium

155 spp. First, a modified approach similar to Blobology, which compares sequence homology, read

156 coverage and GC content, was performed (Kumar, Jones, Koutsovoulos, Clarke, \& Blaxter

157 2013). Contigs from a preliminary genome assembly created with SOAPdenovo2 v0.4

158 (parameters -K $95-\mathrm{R}$ ) were compared for homology against the coral Acropora digitifera

159 (NCBI: GCF_000222465.1) and symbiont Breviolum minutum (OIST: symbB.v1.0.genome.fa) 
161 Shinzato et al. 2011; Shoguchi et al. 2013). The sequence matches to the nt database for contigs

162 that had no match to either coral or symbiont genome, were used to create a local contamination

163 database to further screen the reads (Luo et al. 2015).

Reads were aligned with Bowtie2 v. 2.2.9 (parameters -q-fast; Langmead \& Salzberg 2012) consecutively to the $A$. digitifera mitochondria (KF448535.1), followed by a concatenated set of three Symbiodiniaceae genomes (Symbiodinium microadriaticum, Breviolum minutum,

167 Fugacium; Aranda et al. 2016; Lin et al. 2015; Shoguchi et al. 2013), and the contamination

168 database. This filtering step, however, only aligned $0.28 \%$ of the reads from $A$. cervicornis to the 169 Symbiodiniaceae genomes. Reads that mapped to Symbiodiniaceae genomes $(n=1,004,992)$ were 170 extracted and assembled using SPADES v3.9.1 with a multi-kmer approach $(-\mathrm{k} 21,33,55,77,99)$

171 (Bankevich et al. 2012). The reads that aligned to the contamination database were assembled 172 separately with SPADES v3.9.1 as described above, resulting in three additional contigs that 173 matched Symbiodiniaceae genomes through blast homology.

175 rounds of gap filling using GapCloser v1.12 and scaffolding using both SSPACE v2.0 (rounds 1, 3, and 5) and LINKs v1.8.5 with A. digitifera scaffolds as the "long-reads" (-t 2 -d $3000-\mathrm{k} 25$,

177 round 2, 4 and 6) on alternate rounds (Boetzer \& Pirovano 2014; Luo et al. 2015; Warren et al.

178 2015). After the first three rounds and then each subsequent round, the contigs/scaffolds were

179 partitioned to either coral or symbiont based on the top scoring match (lowest e-value) against a

180 local blast database containing three Symbiodiniaceae genomes and five cnidarian genomes

181 (Hydra, Hydractinia, Nematostella, Exaiptasia, Acropora digitifera). If the scaffolds equally

182 matched cnidarian and symbiont sequences or did not match either they were retained in the

183 coral fraction. Scaffolds identified as Symbiodiniaceae after the six rounds as well as those 
assembled with SPADES above were combined. Two additional rounds of scaffolding with

GapCloser were performed. To remove any remaining sequences matching cnidarian sequences,

187 a final round of scaffold partitioning was performed by comparing the scaffolds against the

188 Acropora spp. genomes: A. digitifera (Shinzato et al. 2011), A. palmata (Kitchen et al.

189 unpublished; http://baumslab.org/research/data), A. cervicornis (Kitchen et al. unpublished), $A$.

190 hyacinthus (Barshis et al. 2013), A. tenuis (Liew, Aranda, \& Voolstra 2016); and Symbiodinium

191 spp. genomes: S. microadriaticum (Aranda et al. 2016), and S. tridacnidorum (Shoguchi et al. 192 2018).

Genome annotation and completeness

Genes were predicted using Augustus v 3.2.3 (Stanke et al. 2006; Stanke, Steinkamp,

196 Waack, \& Morgenstern 2004). Each predicted gene in S. 'fitti' was queried against the NCBI nr, 197 Uniprot SwissProt and trembl databases using blastx 2.6.0+ $(\max$ target seqs $=5$, $\max$ hsps $=1$, 198 e-value = 1e-5; Altschul et al. 1997; Apweiler et al. 2004; Bairoch \& Apweiler 1997; UniProt 199 2014). Gene models were also compared to the $S$. microadriaticum gene and protein predictions 200 (NCBI: GCA_001939145.1) using blast (Altschul et al. 1997). To calculate assembly statistics 201 and compare completeness to other Symbiodiniaceae genomes available at the time of analysis

202 (F. kawagutii, C. goreaui, B. minutum, S. microadriaticum, S. tridacnidorum), we compared our $203 S$. 'fitti' assembly and the aforementioned assemblies using an online version of CEGMA with 204 the eukaryote ortholog set executed by gVolante (https:/gvolante.riken.jp; Simão, Waterhouse, 205 Ioannidis, Kriventseva, \& Zdobnov 2015). Orthofinder v2.2.1 with default settings (Emms \& 
206 Kelly 2015) was used to identify unique and shared orthogroups between S. 'fitti' and six other

207 Symbiodiniaceae species.

208

209

210

211

212

213

214

215

216

217

218

219

220

221

222

223

224

225

226

227

S. 'fitti' infection status

Presence of multiple $S$. 'fitti' strains within a coral host sample was determined using 12 S. 'fitti' specific microsatellite loci as described by Baums et al. (2014). S. 'fitti' is haploid, thus samples with multiple alleles for any given $S$. 'fitti' microsatellite locus were deemed co-infected and removed from downstream analyses (Table S1).

\section{Variant detection and filtering}

For the purpose of SNP analyses, the $S$. 'fitti' genome assembly based on the $A$.

cervicornis metagenome was used as a reference for variant calling of the deeply-sequenced $A$.

palmata and all shallow genome samples (Table S1). The 47 shallow S. 'fitti' and 1 deep

sequenced $A$. palmata "like” S. 'fitti' genome samples were aligned using BWA v0.7.15 (Li

2013). Samtools v1.4 was used to remove PCR duplicates from the BAM file and alignment

statistics were calculated using samtools flagstat (Table S1; Li et al. 2009). Variants were

gathered using samtools mpileup using the -ugAEf and -t AD,DP flags and called using bcftools

v1.4 using the haploid, -f GQ, and -vmO z flags (Li et al. 2009; Narasimhan et al. 2016). The

bcftools (Li et al. 2009; Narasimhan et al. 2016) - m2 -M2 -v snps flags were used to separate

SNPs from the output and the $-\mathrm{v}$ indels flag was used to remove indels from the output

(Narasimhan et al. 2016). High-quality SNPs and indels were characterized as variants with a

quality score over 200 and with no more than $20 \%$ of variant calls missing at a given site among 
228 all samples (Danecek et al. 2011; Narasimhan et al. 2016). Only high-quality SNPs were used in 229 subsequent analyses.

Population structure

The $p s b A$ minicircle was assembled from each sample to determine if the dominant algal partners amongst the three host taxa were all $S$. 'fitti' The psbA minicircle in the $S$. 'fitti'

234 genome assembly was identified through blast searches against three psbA sequences from NCBI $235 \quad(\mathrm{JN} 557866.1=$ Symbiodinium type A3, JX094319.1 $=$ Breviolum minutum, and AJ884898.1=B. faviinorum (Barbrook, Visram, Douglas, \& Howe 2006; Mungpakdee et al. 2014; Pochon,

237 Putnam, Burki, \& Gates 2012). The $p s b A$ minicircle for the remaining samples was assembled 238 using two approaches. In the first approach, filtered and trimmed short-read sequences were 239 mapped to $S$. 'fitti' psbA sequence (scaffold71443) using Bowtie 2 v2.3.4.1 (Langmead \&

240 Salzberg 2012) with the --sensitive mode parameter. Mapped reads were extracted using 241 bedtools v2.26.0 (Quinlan \& Hall 2010) and assembled using SPAdes v3.10.1 (Bankevich et al. 242 2012) with various kmer sizes (-k 21, 33, 55, 77 and 99). In the second approach, the de novo 243 organelle genome assembler NOVOplasty was used (Dierckxsens, Mardulyn, \& Smits 2016).

244 The $S$. 'fitti' genome $p s b A$ sequence was used as the seed sequence to extract similar sequences

245 from the original, unfiltered reads for each sample. A consensus sequence from the two

246 approaches for each sample was created after manual alignment of the sequences using MEGA6

247 (Tamura, Stecher, Peterson, Filipski, \& Kumar 2013). Phylogenomic patterns of $S$. 'fitti' allelic composition were determined using a subset of

249 the high-quality SNPs without missing data with the RAxML-NG v. 0.9.0 GTR+FO+G

250 nucleotide model (Stamatakis 2014). The tree topology with the lowest likelihood score is 
251 presented with nodal support from 100 bootstrap replicates (Stamatakis 2014). Population

252 structure was evaluated using STRUCTURE v2.3.4 for the 58,813 high-quality SNPs (Pritchard,

253 Stephens, \& Donnelly 2000). Additionally, the R package poppr v2.1.0 was used to determine

254 the multilocus genotype of each strain using high quality SNPs with different genetic distance

255 thresholds ranging 10-20\% (See table S1; Kamvar, Tabima, \& Grünwald 2014; Kitchen et al.

256 2020) Clusters in multivariate space were detected using the $p c a$ function in PCAdapt (Luu,

257 Bazin, \& Blum 2017). An Analysis of Molecular Variance (AMOVA, poppr R package) was

258 used for additional detection of population differentiation (Kamvar et al. 2014).

259

Determination of variants under selection

261 Two different methods were used to identify candidate loci under selection. BayeScan v2.1 is a

262 Bayesian method that incorporates uncertainty of allele frequencies between populations with

263 small sample sizes (Fischer, Foll, Excoffier, \& Heckel 2011; Foll, Fischer, Heckel, \& Excoffier

264 2010; Foll \& Gaggiotti 2008). The default BayeScan settings were used for determining SNPs

265 under selection when accounting for $S$. 'fitti' host, location, and host*location interactions.

266 PCAdapt v4.0.3 was used to determine SNPs under selection without prior population

267 information using the default settings (Knaus \& Grünwald 2017). Outliers from BayeScan were

268 determined as markers where FDR $<0.05$ and outliers from PCAdapt v4.0.3 were determined by

269 q-values larger than the alpha value (0.05; Fischer et al. 2011; Foll et al. 2010; Foll \& Gaggiotti

270 2008; Knaus \& Grünwald 2017). Outlier loci with a Bayes probability of 1 and q-value of 0

271 which becomes infinite following logarithmic transformation and were removed from plotting.

272 All statistics from SNPs under selection, their proximity to coding regions, sequence coverage, 


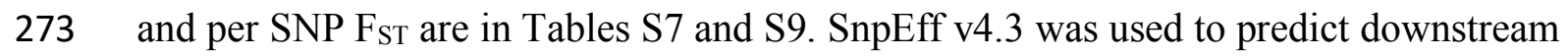

274 functional implications of all detected variants (De Baets et al. 2011).

275

276 Data and code availability

277 Raw data is publicly available on NCBI under SRA project PRJNA473816. Code for data

278 analysis and figure generation is available on github (https://github.com/hgreich/Sfitti).

279

280 Results

281

Genome statistics and comparison to other Symbiodiniaceae

282

The Symbiodinium 'fitti' assembly has a total nucleotide length of over $600 \mathrm{Mb}$

$283(601,782,011 \mathrm{bp})$ and contains 274,185 contigs/scaffolds (A. cervicornis-S. 'fitti' CFL14120; Fig.

284 1, Table S2). The A. palmata- S. 'fitti' (PFL1012) deeply-sequenced sample had 297,371,995

285 reads map to the $A$. cervicornis-S. 'fitti' (CFL14120) reference (19\% mapping rate, 8.5\% paired

286 reads, $4.2 \%$ singleton reads; Table S1). The shallow-sequenced genome samples with one $S$.

287 'fitti' genotype had an average of 6,461,332 reads map to the reference (19.2\% mapping rate,

$2888.8 \%$ paired reads, $4.3 \%$ singleton reads; Table S1). The GC content and number of ambiguous

289 bases were comparable to the $S$. microadriaticum assembly at $50.24 \%$ and $4.82 \%$, respectively

290 (Fig. 1, Table S2; Aranda et al. 2016). The genome completeness was assessed by the

291 identification of the 248 core genes queried using the CEGMA program. The S. 'fitti' assembly

292 had 55 complete proteins, 79 complete + partial proteins, and 169 missing proteins, which is

293 comparable to the other Symbiodiniaceae assemblies queried (Table S2). The average number of

294 orthologs per core gene was $\sim 1.4$ for the Symbiodiniaceae assemblies (Table S2). Gene

295 prediction of $S$. 'fitti' assembly revealed 24,286 gene models, however, many were incomplete 
296 (i.e., missing start or stop codon; Table S2, S3). In the gene family analysis, 3,368 orthogroups

297 were found to be shared by the Symbiodiniaceae assemblies excluding S. 'fitti' whereas 2,982

298 orthogroups were found to be shared by all Symbiodiniaceae assemblies (Fig. S1; including $S$.

299 'fitti'). Additionally, 1,898 orthogroups were uniquely shared by $S$. 'fitti' and its closest relative

300 S. tridacnidorum whereas 1,357 orthogroups were shared by the three assemblies from the genus

301 Symbiodinium (Fig. S1). S. 'fitti' had 11 orthogroups that were not shared with any other

302 Symbiodiniaceae assemblies.

Variation of the allelic composition of Symbiodinium 'fitti'

Based on the analysis of $S$. 'fitti' specific microsatellite loci, the majority of shallowsequenced samples harbored one strain of $S$. 'fitti $(\mathrm{n}=47,75.9 \%$ of $A$. palmata-S. 'fitti' and $82.6 \%$ of $A$. cervicornis- and A. prolifera-S. 'fitti'; Table S1) and were used for further analysis. A total of 2,505,230 SNPs and 569,337 indels were identified between all samples. Of these, 58,538 SNPs and 1,874 indels were considered high-quality (Fig. 1). The 58,538 high-quality SNPs represent a range of per SNP fixation levels from 0 to 1 (Fig. S2). The Transition:

311 Transversion ratio of the high-quality SNPs was 1.79 and did not vary by host species (Table

312 S1). Of the high-quality SNPs, 12,780 (21.8\%) occurred in coding regions (Table S4). The 313 majority of the SNPs $(87.5 \%)$ in coding regions matched other Symbiodiniaceae genomic 314 resources (primarily the closely related species, S. microadriaticum). Multi-locus genotype 315 (MLG) filtering of the 58,538 "high quality" SNPs indicated each sample represented a unique 316 MLG (strain), consistent with the microsatellite analysis, and was retained for downstream 317 analyses (Table S1). Additional filtering to remove variants with missing data resulted in 6,813 318 high-quality SNPs, hereafter called conservative SNPs. After this procedure of quality filtering 
319 SNPs and setting a stringent missing data threshold, the average read coverage increased from 1.53 to 11.3 per SNP (Table S1; average $655.3 \%$ increase).

Patterns of host-specificity and biogeography within S. 'fitti' between symbiont strains with respect to their host taxa, confirming that $S$. 'fitti' is one species species level $(86.1+\%$; Table S5). Variation attributed to host species explained $11.6 \%$ of the components of covariance $($ sigma $=87.0)$ and then variation among the geographic location of each host explained $2.3 \%$ of the components of covariance ( sigma $=17.1$; Table S5). Variation among geographic locations explained a negative amount of the components of covariance ($5.4 \%$, sigma $=-39.3$ ) whereas variation among the host taxa at the various geographic locations explained $16.3 \%$ of the components of covariance $($ sigma $=117.6$; Table S5). than geographic origins in a Principal Component Analysis (PCA) with the high-quality SNPs

334 and in the Maximum Likelihood tree with the conservative SNPs (Figs. 2, 3). In the PCA, 16\% 335 of variance was explained by PC1 whereas $13.6 \%$ was explained by PC2 (Fig. 2). Within each 336 host, there was some indication of biogeographic partitioning in the phylogeny but not in the 337 PCA (Figs. 2, 3; Table S6). The $S$. 'fitti' associated with $A$. prolifera were found intermediate to 338 the parental species in the Maximum Likelihood tree and clustered loosely together but were more similar to the A. palmata S. 'fitti' (Fig. 2). Analysis of STRUCTURE output using the

340 delta $K$ method (Evanno, Regnaut, \& Goudet 2005) identified three clusters as the most likely K

341 (Table S6). The three clusters largely corresponded to host taxa (Fig. 4). 
SNPs under selection

Of the high-quality SNPs, 4,987 (8.5\%) were determined as selection outliers by

345 PCAdapt (Fig. 5). When BayeScan accounted for host identity, location of host, and host by

346 location interaction, 217, 5 and 197 selection outliers were identified, respectively ( $\mathrm{n}=370$ SNPs;

347 Fig. 5; Table S7). Additionally, 339 selection outlier SNPs were shared between the two

348 programs (Fig. 5; Table S7). 103 outlier loci identified by BayeScan had a Bayes probability of 1

349 and q-value of 0 which becomes infinite following logarithmic transformation and were therefore

350 removed from the Manhattan plot (Fig. 5) but were reported in table S7. For each set of SNPs

351 under selection, a subset was found in coding regions (899 from PCAdapt, 19 from BayeScan, 14

352 from both callers; Table S7). The 14 outliers in coding regions that were shared by both callers

353 were found in the coding regions of Putative cytosolic oligopeptidase, tankyrase-like proteins,

354 alpha-agarase, and uncharacterized proteins (Table S7).

355

Predicted functional implications of SNPs within S. 'fitti'

357

Of all high-quality SNPs, SnpEff identified 60,373 modifier/non-coding variants

$358(84.43 \%), 3,629$ moderate/mostly harmless variants $(5.08 \%), 7,451$ low impact variants that

359 might change protein efficiency/effectiveness (10.42\%), and 51 highly disruptive SNPs $(0.07 \%)$.

360 Of the predicted mutations, SnpEff identified 3,644 non-synonymous mutations (32.87\%), 32

361 premature stop codon/nonsense mutations $(0.29 \%)$, and 7,410 synonymous mutations $(66.84 \%$;

362 Table S8). The aforementioned mutations are predicted to cause 11,086 codon changes and 3,676

363 amino acid changes (Tables S8, S9). SnpEff predicted the 14 aforementioned outlier mutations 
364 of Putative cytosolic oligopeptidase, tankyrase-like proteins, and alpha-agarase as modifier

365 variants found in the introns (Tables S7-S10).

\section{Discussion}

The population dynamics and evolutionary history of reef-building corals are relatively well studied compared to the dinoflagellate symbionts they harbor. However, selection acts on both partners and differences in life-history characteristics between algae and corals suggest that

371 the spatial and temporal scale of adaptation may differ. This would have consequences for our 372 understanding of how they may adapt to rapidly changing climates. Genomic data of Caribbean

373 acroporids reveals fine-scale population structure within the host taxa (Devlin-Durante \& Baums

374 2017; Kitchen et al. 2019; Kitchen et al. 2020). Previous analyses of microsatellite loci

375 demonstrated that $S$. 'fitti' gene-flow scales were smaller than its host Acropora palmata (Baums

376 et al. 2014), however, this study jointly analyzed SNP data from all three Caribbean acroporid

377 host taxa. We showed that the allelic composition of sympatric $S$. 'fitti' populations are

378 partitioned by host taxon and describe two potential scenarios that would lead to this result (Figs.

379 2-4). Differentiation of $S$. 'fitti' by host taxon implies partner selectivity, which may be the result

380 of coevolution (Scenario 1). Alternatively, coevolution and partner selectivity per se (i.e. via

381 specific recognition of genetic variants among partners) may not explain the patterns of allelic

382 variation. Differences in the micro-environment (light, depth, nutrient availability) associated

383 with the habitat preferences of the host taxa may drive symbiont differentiation without specific

384 recognition interactions or coevolution (Scenario 2). However, it is likely a combination of these

385 mechanisms that drives the coevolution of S. 'fitti' strains within Caribbean acroporids. In either

386 case, $S$. 'fitti' genetic diversity is tied to that of its endangered hosts. 
Host selectivity of symbiont strains in a horizontal symbiont transmission

Broadcast spawning coral species acquire symbiotic partners horizontally each generation spawning corals and their symbionts is commonly observed at the species level with multimarker and microsatellite approaches (Chan et al. 2019; LaJeunesse 2001; Lewis et al. 2019).

Here, we add population genomic analyses using SNP data to reveal that sub-species level partitioning occurs between $S$. 'fitti' and its three Caribbean acroporid hosts (Figs. 2-4). Though

401 adult colonies of Caribbean acroporids primarily associate with $S$. 'fitti', they can occasionally harbor other genera of Symbiodiniaceae (Breviolum spp., Durusdinium trenchii and

Cladocopium spp.), but these associations are often transitory and revert to S. 'fitti' over time

404 (Baums, Johnson, Devlin-Durante, \& Miller 2010; Thornhill, LaJeunesse, Kemp, Fitt, \& Schmidt 405 2006b). modulated by cell recognition pathways such as lectin-glycan interactions and might contribute 
410 Logan, LaFlamme, Weis, \& Davy 2010; Parkinson et al. 2018; Weis, Reynolds, deBoer, \&

411 Krupp 2001; Wood-Charlson, Hollingsworth, Krupp, \& Weis 2006). Though the exact role of

412 cell-signaling in host selectivity has yet to be fully described, the specificity between partners is

413 maintained by specialization pressures (LaJeunesse et al. 2018; Parkinson et al. 2018; Wood-

414 Charlson et al. 2006). The $\sim 160$ million years following the widespread adaptive radiation of

415 stony corals and Symbiodiniaceae has resulted in their current inter-dependence on one another

416 (LaJeunesse et al. 2018). Consequently, the various symbiotic pairings remain adapted to meet

417 the unique biochemical and metabolic demands of each host microenvironment (Barott, Venn,

418 Perez, Tambutté, \& Tresguerres 2015; Sogin, Anderson, Williams, Chen, \& Gates 2014). The

419 different morphologies and corallite structures possessed by each acroporid results in different

420 light scattering properties and ultimately, light availability to the resident endosymbiont

421 (Enríquez et al. 2017). These differences likely lead to strong selection pressures unique to each

422 acroporid taxa. Ultimately, the specificity between $S$. 'fitti' strains and their acroporid hosts

423 could be maintained by its ability (or lack thereof) to meet the metabolic needs of its host and

424 adapt to the microenvironment created by its host.

425

426 The role of symbiont selectivity as a driver of S. 'fitti' intraspecific genomic variation

427 Akin to role of host selectivity, that of the symbiont is also important for upholding

428 partner specificity in symbioses. The diversity and abundance of Symbiodiniaceae in the water

429 column is not necessarily proportional to their counterparts partaking in mutualisms with reef-

430 building corals (Cunning, Yost, Guarinello, Putnam, \& Gates 2016; Littman, van Oppen, \&

431 Willis 2008; Manning \& Gates 2008). However, some lineages of Symbiodiniaceae possess the

432 ability to establish symbiosis with non-coral invertebrates (Cunning et al. 2016; Decelle et al. 
433 2018). The total diversity of $S$. 'fitti' likely spans beyond strains that inhabit acroporids and may

434 incorporate free-living conspecifics living in the water column, sediments, etc. Therefore, the

435 slight differences in allelic composition of each $S$. 'fitti' strain may be a result of the differential

436 host preference of the available symbionts (Figs. 2-4). However, symbiotic $S$. 'fitti' strains might

437 be attracted to the different microbial composition and abundance in the water column adjacent

438 to a coral colony that constitute the 'ecosphere' surrounding each acroporid (Weber, González-

439 Díaz, Armenteros, \& Apprill 2019). Similarly, the food associated with each 'ecospheres’ may

440 attract different Symbiodiniaceae (Pollock et al. 2018; Weber et al. 2019). Putative intraspecific

441 variation in the swimming availability and chemosensory responses of S. 'fitti' may also, in part,

442 dictate which Symbiodiniaceae persist in each ecosphere (Fitt 1984; Fitt 1985; Fitt, Chang, \&

443 Trench 1981; Kamykowski, Reed, \& Kirkpatrick 1992). Future experimental validation of

444 intraspecific variation in S. 'fitti' swimming and chemosensory ability and how it pertains to

445 selectivity of their acroporid hosts (and 'ecospheres') will shed light on how inter-partner

446 specificity is maintained.

447

Coevolution as a driver of S. 'fitti' intraspecific genomic variation

Coevolution is the process by which two interacting species reciprocally adapt to each

450 other (sensu Janzen 1980). The coevolution of cnidarian-dinoflagellate mutualisms, in part, has

451 resulted in the long-term fidelity between partners (Figs. 2-4; LaJeunesse et al. 2018; Stanley

452 2006). Though the fossil record supports the coevolution of these mutualisms (Muscatine et al.

453 2005; Stanley 2006), experimental follow up and verification has received far less attention.

454 Advances in phylogenomics reveal extensive differentiation of genomic features and gene family

455 enrichment when comparing symbiotic and free-living Symbiodinium spp., a potential biproduct 
456 of coevolving with their hosts (González-Pech, Bhattacharya, Ragan, \& Chan 2019a; González-

457 Pech, Ragan, \& Chan 2017; González-Pech et al. 2019b). Similarly, population genetic analyses

458 of host and symbiont reveals widespread long-term partnerships between several cnidarian

459 species and their endosymbiotic dinoflagellates, which might be the biproduct of coevolution

460 (Baums et al. 2014; O’Donnell et al. 2018; Poland \& Coffroth 2017; Thornhill et al. 2006a;

461 Thornhill et al. 2017; Thornhill et al. 2006b). Therefore, the correspondence of the allelic

462 variation of $S$. 'fitti' to its host acroporids is likely, in part, the result of coevolution. The

463 STAGdb genotyping array (SNPchip) can be harnessed to experimentally verify the

464 contributions of coevolution, host specificity, and symbiont selectivity to the evolutionary

465 dynamics of Acropora-S. 'fitti' symbioses' (Kitchen et al. 2020).

466

467 The special case of the F1 coral hybrid as habitat

468 The shared history ( 2.6-3.6 million years of coexistence) between parents $A$. palmata

469 and $A$. cervicornis with their symbiont, S. 'fitti' may have allowed sufficient time for co-

470 evolutionary processes to play out (and so may help explain the strain differentiation of $S$. 'fitti'

471 by host; Figs 2-4; Budd \& Johnson 1999; McNeill et al. 1997). However, the situation differs for

472 their first-generation hybrid, A. prolifera, which cannot directly respond to selection pressure

473 from $S$. 'fitti' via differential successful sexual reproduction of its colonies because most are

474 sterile (Vollmer \& Palumbi 2002). Thus, any changes in the allele frequencies of $A$. prolifera are

475 restricted to somatic mutations occurring within their lifetime which can be on the order of

476 hundreds of years (Irwin et al. 2017). Though the fossil record of $A$. prolifera is rather sparse

477 (McNeill et al. 1997), S. 'fitti' may have encountered these colonies over many thousands of 
478 years as they are generated anew with each hybridization event. Thus, while a host co-adaptive 479 response is unlikely, S. 'fitti' may have evolved strains that preferentially colonize A. prolifera.

Environmental differentiation as a driver of intraspecific genomic variation

Burdon, \& Bever 2007). S. 'fitti' population dynamics are confounded with differences in host habitat preferences including light, temperature, nutrient concentration, and food availability in the water column (Crossland \& Barnes 1983; Miller 1995; Terraneo et al. 2019; Williams et al. 2018). The inverse relationship between depth and light availability is a common driver of coral and Symbiodiniaceae zonation (Bongaerts et al. 2015a; Bongaerts et al. 2015b; Bongaerts et al. 2017; Fogarty 2012; Goulet, Lucas, \& Schizas 2019; LaJeunesse 2002; Serrano et al. 2014; Serrano et al. 2016). Throughout much of their distribution, Caribbean acroporid species reside

490 in different habitats (Fogarty 2012; Goreau 1959). Specifically, A. cervicornis occupies a lower 491 light habitat ( $\sim 10 \mathrm{~m}$ depth) relative to its high-light dwelling ( $\sim 3 \mathrm{~m}$ depth) sibling species $A$.

492 palmata (Fogarty 2012; Goreau 1959; LaJeunesse 2002). Although the hybrid's depth 493 distribution often overlaps with A. palmata, it can also be found in less than $1 \mathrm{~m}$ of water 494 (Fogarty 2012). Therefore, adaptation to different light availabilities may correspond to genomic 495 differentiation between the shallow A. palmata- $S$. 'fitti' and A. prolifera- $S$. 'fitti' versus deep $A$. 496 cervicornis- S. 'fitti' (Figs. 2-4; Finney et al. 2010; Kirk, Andras, Harvell, Santos, \& Coffroth 497 2009). Microenvironments created by light attenuation at depth may lead to range-limited 498 dispersal of $S$. 'fitti' and modulate the available pool of symbionts (Finney et al. 2010; Serrano et 499 al. 2016). Furthermore, the differences in the skeletal morphology of acroporids result in 500 different light scattering properties that likely have profound effects on the light availability and 
501 microenvironments for their resident $S$. 'fitti' (Enríquez et al. 2017; Gladfelter 1983; Gladfelter

502 2007). The different light regimes, skeletal features and flow fields associated with each

503 acroporid may exert some selection pressure on symbiont strains.

504

505 Genomic basis for extended phenotypes in S. 'fitti' - acroporid symbiosis

506 The physiological capacity of the holobiont (coral and symbiont) hinges upon the specific

507 partner pairings as well as external (environmental) drivers (Parkinson \& Baums 2014). The

508 large number of non-synonymous SNPs differentiating the S. 'fitti' strains among their hosts may

509 demarcate the onset of eventual speciation (Fig. 5; Tables S7-10) although it is difficult to know

510 what barriers to gene flow may exist that allow for such a process. Changes in amino acid

511 sequences and protein efficiency resulting from these mutations may serve as the genomic basis

512 causing intraspecific variation in physiological aptitude (Parkinson et al. 2015a; Parkinson \&

513 Baums 2014). Similarly, the candidate genes under selection identified by BayeScan and

514 PCAdapt may underlie the strain differentiation by host species (Tables S7-10). Non-

515 synonymous mutations in putative cytosolic oligopeptidase and alpha-agarase regions may result

516 in the subtle alteration of zinc and calcium ion binding, respectively, which in turn likely

517 contribute to variation in the physiological capacity of S. 'fitti' (Table S7-S10; Kmiec, Teixeira,

518 Murcha, \& Glaser 2016; Zhang et al. 2018). Further, these genotypic and phenotypic differences

519 may facilitate the adaptation of each $S$. 'fitti' strain to the internal and external

520 microenvironments associated with each host niche and meeting their metabolic and nutritional

521 demands (Figs. 2-5; Tables S7-10; Hemond, Kaluziak, \& Vollmer 2014; Muscatine, Porter, \&

522 Kaplan 1989; Reich, Rodriguez, LaJeunesse, \& Ho 2020; Sogin et al. 2014). 
Conclusion

525

We show here that the population genetic structure of Symbiodinium 'fitti' is, in part,

526 explained by its host association. Because the host species occupy different habitats, we cannot

527 yet disentangle the role of host versus depth as a potential driver of population genetic structure.

528 However, the genomic resources for the $S$. 'fitti' - acroporid system described here can be used in

529 future studies to determine whether, and to what degree, the observed variation of allelic

530 composition is a result of host selectivity, symbiont selectivity, coevolution, environmental

531 differentiation, or a combination of these mechanisms. The appreciation of population genetic

532 structure and evolutionary dynamics of both coral holobiont partners will better inform the

533 genomic underpinnings of their phenotypes and physiological capacity.

\section{4}

\section{Acknowledgements}

536 We thank the PSU genomics facility for assistance with library prep and sequencing. We thank

537 Prof. Todd LaJeunesse for assistance with the $p s b A$ phylogeny. Funding for this project was

538 supported by NSF-OCE-1537959 (to IBB) and NSF-OCE-1538469 (to NDF). HGR was

539 supported through NSF-OCE-1636022 (to T. LaJeunesse). Permits for samples include Florida:

540 CRF permit numbers CRF-2017-009, CRF-2017-012, NOAA FKNMS permit numbers FKNMS-

541 2011-159-A4, FKNMS-2001-009, FKNMS-2014-148-A2, and FKNMS-2010-130-A, Belize:

542 CITES Permit 0385, 7487 and 7488; Curacao: CITES Permit 16US784243/9 and

543 12US784243/9; and USVI Department of planning and natural resources, Division of fish and

544 wildlife DFW14017T.

\section{5}

546

\section{References}


Abrego D., Van Oppen M., \& Willis B. L. (2009) Onset of algal endosymbiont specificity varies among closely related species of Acropora corals during early ontogeny. Molecular ecology 18, 3532-3543.

Altschul S. F., Madden T. L., Schäffer A. A., Zhang J., Zhang Z., Miller W., \& Lipman D. J. (1997) Gapped BLAST and PSI-BLAST: a new generation of protein database search programs. Nucleic acids research 25, 3389-3402.

Apweiler R., Bairoch A., Wu C. H., Barker W. C., Boeckmann B., Ferro S., . . Magrane M. (2004) UniProt: the universal protein knowledgebase. Nucleic acids research 32, D115D119.

557

558

Aranda M., Li Y., Liew Y. J., Baumgarten S., Simakov O., Wilson M. C., . . Voolstra C. R. (2016) Genomes of coral dinoflagellate symbionts highlight evolutionary adaptations conducive to a symbiotic lifestyle. Scientific reports $\mathbf{6}, 39734$.

Baird A. H., Guest J. R., \& Willis B. L. (2009) Systematic and biogeographical patterns in the reproductive biology of scleractinian corals. Annual Review of Ecology, Evolution, and Systematics 40, 551-571.

Bairoch A., \& Apweiler R. (1997) The SWISS-PROT protein sequence data bank and its supplement TrEMBL. Nucleic acids research 25, 31-36.

Baker K. G., Radford D. T., Evenhuis C., Kuzhiumparam U., Ralph P. J., \& Doblin M. A. (2018) Thermal niche evolution of functional traits in a tropical marine phototroph. Journal of Phycology 54, 799-810.

Bankevich A., Nurk S., Antipov D., Gurevich A. A., Dvorkin M., Kulikov A. S., ... Prjibelski A. D. (2012) SPAdes: a new genome assembly algorithm and its applications to singlecell sequencing. Journal of Computational Biology 19, 455-477. 
571 Barbrook A. C., Visram S., Douglas A. E., \& Howe C. J. (2006) Molecular Diversity of

572

573

574

575

576

577

578

579

580

581

582

583

584

585

586

587

588

589

590

591

592

593

Dinoflagellate Symbionts of Cnidaria: The psbA Minicircle of Symbiodinium. Protist 157, 159-171.

Barneah O., Weis V. M., Perez S., \& Benayahu Y. (2004) Diversity of dinoflagellate symbionts in Red Sea soft corals: mode of symbiont acquisition matters. Marine Ecology Progress Series 275, 89-95.

Barott K. L., Venn A. A., Perez S. O., Tambutté S., \& Tresguerres M. (2015) Coral host cells acidify symbiotic algal microenvironment to promote photosynthesis. Proceedings of the National Academy of Sciences 112, 607-612.

Barshis D. J., Ladner J. T., Oliver T. A., Seneca F. O., Traylor-Knowles N., \& Palumbi S. R. (2013) Genomic basis for coral resilience to climate change. Proceedings of the National Academy of Sciences 110, 1387-1392.

Baums I. B., Baker A. C., Davies S. W., Grottoli A. G., Kenkel C. D., Kitchen S. A., . . Shantz A. A. (2019) Considerations for maximizing the adaptive potential of restored coral populations in the western Atlantic. Ecological Applications 0, e01978.

Baums I. B., Devlin-Durante M. K., \& LaJeunesse T. C. (2014) New insights into the dynamics between reef corals and their associated dinoflagellate endosymbionts from population genetic studies. Molecular ecology 23, 4203-4215.

Baums I. B., Johnson M. E., Devlin-Durante M. K., \& Miller M. W. (2010) Host population genetic structure and zooxanthellae diversity of two reef-building coral species along the Florida Reef Tract and wider Caribbean. Coral Reefs 29, 835-842.

Baums I. B., Miller M. W., \& Hellberg M. E. (2005) Regionally isolated populations of an imperiled Caribbean coral, Acropora palmata. Molecular ecology 14, 1377-1390. 
Baums I. B., Miller M. W., \& Hellberg M. E. (2006) Geographic variation in clonal structure in a reef-building Caribbean coral, Acropora palmata. Ecological Monographs 76, 503-519.

Bellantuono A. J., Dougan K. E., Granados-Cifuentes C., \& Rodriguez-Lanetty M. (2019) Freeliving and symbiotic lifestyles of a thermotolerant coral endosymbiont display profoundly distinct transcriptomes under both stable and heat stress conditions. Molecular ecology

600

601

602

603

604

605

606

607

608

609

610

611

612

613

614

615

$$
\text { 28, 5265-5281. }
$$

Boetzer M., \& Pirovano W. (2014) SSPACE-LongRead: scaffolding bacterial draft genomes using long read sequence information. BMC Bioinformatics 15, 211.

Bongaerts P., Carmichael M., Hay K. B., Tonk L., Frade P. R., \& Hoegh-Guldberg O. (2015a) Prevalent endosymbiont zonation shapes the depth distributions of scleractinian coral species. Royal Society open science 2, 140297.

Bongaerts P., Frade P. R., Hay K. B., Englebert N., Latijnhouwers K. R., Bak R. P., .. . HoeghGuldberg O. (2015b) Deep down on a Caribbean reef: lower mesophotic depths harbor a specialized coral-endosymbiont community. Scientific reports $\mathbf{5}, 7652$.

Bongaerts P., Riginos C., Brunner R., Englebert N., Smith S. R., \& Hoegh-Guldberg O. (2017) Deep reefs are not universal refuges: Reseeding potential varies among coral species. Science Advances 3.

Budd A. F., \& Johnson K. G. (1999) Origination preceding extinction during late Cenozoic turnover of Caribbean reefs. Paleobiology 25, 188-200.

Buerger P., Alvarez-Roa C., Coppin C. W., Pearce S. L., Chakravarti L. J., Oakeshott J. G., ... van Oppen M. J. H. (2020) Heat-evolved microalgal symbionts increase coral bleaching tolerance. Science Advances 6, eaba2498. 
616

617

618

619

620

621

622

623

624

625

626

627

628

629

630

631

632

633

634

635

636

637

638

Chakravarti L. J., \& van Oppen M. J. H. (2018) Experimental Evolution in Coral

Photosymbionts as a Tool to Increase Thermal Tolerance. Frontiers in Marine Science 5, 227.

Chan A. N., Lewis C. L., Neely K. L., \& Baums I. B. (2019) Fallen Pillars: The Past, Present, and Future Population Dynamics of a Rare, Specialist Coral-Algal Symbiosis. Frontiers in Marine Science 6, 218.

Chi J., Parrow M. W., \& Dunthorn M. (2014) Cryptic Sex in Symbiodinium (Alveolata, Dinoflagellata) is Supported by an Inventory of Meiotic Genes. Journal of Eukaryotic Microbiology 61, 322-327.

Coffroth M. A., Goulet T. L., \& Santos S. R. (2001) Early ontogenic expression of specificity in a cnidarian-algal symbiosis. Mar Ecol Prog Ser 222.

Crossland C. J., \& Barnes D. J. (1983) Dissolved nutrients and organic particulates in water flowing over coral reefs at Lizard Island. Marine and Freshwater Research 34, 835-844.

Cunning R., Gillette P., Capo T., Galvez K., \& Baker A. (2015) Growth tradeoffs associated with thermotolerant symbionts in the coral Pocillopora damicornis are lost in warmer oceans. Coral Reefs 34, 155-160.

Cunning R., Yost D. M., Guarinello M. L., Putnam H. M., \& Gates R. D. (2016) Variability of Symbiodinium Communities in Waters, Sediments, and Corals of Thermally Distinct Reef Pools in American Samoa. PloS one 10, e0145099.

Cziesielski M. J., Schmidt-Roach S., \& Aranda M. (2019) The past, present, and future of coral heat stress studies. Ecology and Evolution 9, 10055-10066.

Danecek P., Auton A., Abecasis G., Albers C. A., Banks E., DePristo M. A., .. . Sherry S. T. (2011) The variant call format and VCFtools. Bioinformatics 27, 2156-2158. 
Davy S. K., Allemand D., \& Weis V. M. (2012) Cell Biology of Cnidarian-Dinoflagellate Symbiosis. Microbiology and Molecular Biology Reviews 76, 229-261.

De Baets G., Van Durme J., Reumers J., Maurer-Stroh S., Vanhee P., Dopazo J., . . Rousseau F. (2011) SNPeffect 4.0: on-line prediction of molecular and structural effects of proteincoding variants. Nucleic acids research 40, D935-D939.

Decelle J., Carradec Q., Pochon X., Henry N., Romac S., Mahé F., . . . de Vargas C. (2018) Worldwide Occurrence and Activity of the Reef-Building Coral Symbiont Symbiodinium in the Open Ocean. Current Biology 28, 3625-3633.e3623.

Devlin-Durante M. K., \& Baums I. B. (2017) Genome-wide survey of single-nucleotide polymorphisms reveals fine-scale population structure and signs of selection in the threatened Caribbean elkhorn coral, Acropora palmata. PeerJ 5, e4077.

Dierckxsens N., Mardulyn P., \& Smits G. (2016) NOVOPlasty: de novo assembly of organelle genomes from whole genome data. Nucleic Acids Research 45, e18-e18.

Emms D. M., \& Kelly S. (2015) OrthoFinder: solving fundamental biases in whole genome comparisons dramatically improves orthogroup inference accuracy. Genome Biology 16, 157.

Enríquez S., Méndez E. R., Hoegh-Guldberg O., \& Iglesias-Prieto R. (2017) Key functional role of the optical properties of coral skeletons in coral ecology and evolution. Proceedings of the Royal Society B: Biological Sciences 284, 20161667.

Evanno G., Regnaut S., \& Goudet J. (2005) Detecting the number of clusters of individuals using the software STRUCTURE: a simulation study. Molecular ecology 14, 2611-2620.

Finney J. C., Pettay D. T., Sampayo E. M., Warner M. E., Oxenford H. A., \& LaJeunesse T. C. (2010) The Relative Significance of Host-Habitat, Depth, and Geography on the 
Ecology, Endemism, and Speciation of Coral Endosymbionts in the Genus

663 Symbiodinium. Microbial Ecology 60, 250-263.

664 Fischer M. C., Foll M., Excoffier L., \& Heckel G. (2011) Enhanced AFLP genome scans detect 665 local adaptation in high-altitude populations of a small rodent (Microtus arvalis). Molecular ecology 20, 1450-1462.

667 Fitt W. K. (1984) The role of chemosensory behavior of Symbiodinium microadriaticum, intermediate hosts, and host behavior in the infection of coelenterates and molluscs with

670

671

672

673 674

675

676

677

678

679 680

681

682 zooxanthellae. Marine Biology 81, 9-17.

Fitt W. K. (1985) CHEMOSENSORY RESPONSES OF THE SYMBIOTIC DINOFLAGELLATE SYMBIODINIUM MICROADRIATICUM (DINOPHYCEAE)1. Journal of Phycology 21, 62-67.

Fitt W. K., Chang S. S., \& Trench R. K. (1981) Motility patterns of different strains of the symbiotic dinoflagellate Symbiodinium (= Gymnodinium) microadriaticum (Freudenthal) in culture. Bulletin of Marine Science 31, 436-443.

Fitt W. K., \& Trench R. K. (1983) The relation of diel patterns of cell division to diel patterns of motility in the symbiotic dinoflagellate Symbiodinium microadria ticum Freudenthal in culture. New Phytologist 94, 421-432.

Fogarty N. D. (2012) Caribbean acroporid coral hybrids are viable across life history stages. Marine Ecology Progress Series 446, 145-159.

Foll M., Fischer M. C., Heckel G., \& Excoffier L. (2010) Estimating population structure from AFLP amplification intensity. Molecular ecology 19, 4638-4647. 
683 Foll M., \& Gaggiotti O. (2008) A Genome-Scan Method to Identify Selected Loci Appropriate for Both Dominant and Codominant Markers: A Bayesian Perspective. Genetics 180, 977-993.

686 Gladfelter E. H. (1983) Skeletal development inAcropora cervicornis. Coral Reefs 2, 91-100.

687 Gladfelter E. H. (2007) Skeletal development in Acropora palmata (Lamarck 1816): a scanning electron microscope (SEM) comparison demonstrating similar mechanisms of skeletal of Coral Reef Symbionts as Intracellular Residents. Trends in Ecology \& Evolution.

González-Pech R. A., Ragan M. A., \& Chan C. X. (2017) Signatures of adaptation and symbiosis in genomes and transcriptomes of Symbiodinium. Scientific reports 7, 15021.

González-Pech R. A., Stephens T. G., Chen Y., Mohamed A. R., Cheng Y., Burt D. W., ... between symbiotic and free-living \&lt;em\&gt;Symbiodinium\&lt;/em\&gt. bioRxiv, 783902. Series 321, 1-7.

Goulet T. L., Lucas M. Q., \& Schizas N. V. (2019) Symbiodiniaceae Genetic Diversity and 
Hemond E. M., Kaluziak S. T., \& Vollmer S. V. (2014) The genetics of colony form and function in Caribbean Acropora corals. BMC Genomics 15, 1133.

708

709

710

711

712

713

714

715

716

717

718

719

720

721

722

723

724

725

726

727

Hemond E. M., \& Vollmer S. V. (2010) Genetic diversity and connectivity in the threatened staghorn coral (Acropora cervicornis) in Florida. PloS one 5, e8652-e8652.

Irwin A., Greer L., Humston R., Devlin-Durante M., Cabe P., Lescinsky H., . . Baums I. B. (2017) Age and intraspecific diversity of resilient Acropora communities in Belize. Coral Reefs 36, 1111-1120.

Janzen D. H. (1980) When is it coevolution?

Kamvar Z. N., Tabima J. F., \& Grünwald N. J. (2014) Poppr: an R package for genetic analysis of populations with clonal, partially clonal, and/or sexual reproduction. PeerJ 2, e281.

Kamykowski D., Reed R. E., \& Kirkpatrick G. J. (1992) Comparison of sinking velocity, swimming velocity, rotation and path characteristics among six marine dinoflagellate species. Marine Biology 113, 319-328.

Kemp D. W., Thornhill D. J., Rotjan R. D., Iglesias-Prieto R., Fitt W. K., \& Schmidt G. W. (2015) Spatially distinct and regionally endemic Symbiodinium assemblages in the threatened Caribbean reef-building coral Orbicella faveolata. Coral Reefs 34, 535-547.

Kirk N. L., Andras J. P., Harvell C. D., Santos S. R., \& Coffroth M. A. (2009) Population structure of Symbiodinium sp. associated with the common sea fan, Gorgonia ventalina, in the Florida Keys across distance, depth, and time. Marine Biology 156, 1609-1623.

Kitchen S. A., Ratan A., Bedoya-Reina O. C., Burhans R., Fogarty N. D., Miller W., \& Baums I. B. (2019) Genomic Variants Among Threatened Acropora Corals. G3: Genes|Genomes|Genetics 9, 1633. 
728 Kitchen S. A., Von Kuster G., Kuntz K. L. V., Reich H. G., Miller W., Griffin S., . . Baums I.

729

730

731

732

733

734

735

736

737

738

739

740

741

742

743

744

745

746

747

748

749

750

B. (2020) STAGdb: a 30K SNP genotyping array and Science Gateway for Acropora corals and their dinoflagellate symbionts. Scientific reports 10, 12488.

Kmiec B., Teixeira P. F., Murcha M. W., \& Glaser E. (2016) Divergent evolution of the M3A family of metallopeptidases in plants. Physiologia Plantarum 157, 380-388.

Knaus B. J., \& Grünwald N. J. (2017) vcfr: a package to manipulate and visualize variant call format data in R. Molecular Ecology Resources 17, 44-53.

Kumar S., Jones M., Koutsovoulos G., Clarke M., \& Blaxter M. (2013) Blobology: exploring raw genome data for contaminants, symbionts and parasites using taxon-annotated GCcoverage plots. Frontiers in Genetics 4, 237.

LaJeunesse T. C. (2001) Investigating the biodiversity, ecology, and phylogeny of endosymbiotic dinoflagellates in the genus Symbiodinium using the ITS region: in search of a "species" level marker. Journal of Phycology 37, 866-880.

LaJeunesse T. C. (2002) Diversity and community structure of symbiotic dinoflagellates from Caribbean coral reefs. Marine Biology 141, 387-400.

LaJeunesse T. C., Parkinson J. E., Gabrielson P. W., Jeong H. J., Reimer J. D., Voolstra C. R., \& Santos S. R. (2018) Systematic Revision of Symbiodiniaceae Highlights the Antiquity and Diversity of Coral Endosymbionts. Current Biology.

LaJeunesse T. C., Thornhill D. J., Cox E. F., Stanton F. G., Fitt W. K., \& Schmidt G. W. (2004) High diversity and host specificity observed among symbiotic dinoflagellates in reef coral communities from Hawaii. Coral Reefs 23, 596-603.

Langmead B., \& Salzberg S. L. (2012) Fast gapped-read alignment with Bowtie 2. Nature methods 9, 357. 
751 Lee S. Y., Jeong H. J., Kang N. S., Jang T. Y., Jang S. H., \& Lajeunesse T. C. (2015)

752

753

754

755

756

757

758

759

760

761

762

763

764

765

766

767

768

769

770

771

772

Symbiodinium tridacnidorum sp. nov., a dinoflagellate common to Indo-Pacific giant clams, and a revised morphological description of Symbiodinium microadriaticum Freudenthal, emended Trench \& Blank. European Journal of Phycology 50, 155-172.

Levin R. A., Beltran V. H., Hill R., Kjelleberg S., McDougald D., Steinberg P. D., \& van Oppen M. J. H. (2016) Sex, scavengers, and chaperones: transcriptome secrets of divergent symbiodinium thermal tolerances. Molecular biology and evolution, msw119.

Lewis A. M., Chan A. N., \& LaJeunesse T. C. (2019) New Species of Closely Related Endosymbiotic Dinoflagellates in the Greater Caribbean have Niches Corresponding to Host Coral Phylogeny. Journal of Eukaryotic Microbiology.

Li H. (2013) Aligning sequence reads, clone sequences and assembly contigs with BWA-MEM. arXiv preprint arXiv:1303.3997.

Li H., Handsaker B., Wysoker A., Fennell T., Ruan J., Homer N., . . . Genome Project Data Processing S. (2009) The Sequence Alignment/Map format and SAMtools. Bioinformatics 25, 2078-2079.

Liew Y. J., Aranda M., \& Voolstra C. R. (2016) Reefgenomics.Org - a repository for marine genomics data. Database $\mathbf{2 0 1 6 .}$

Lin S., Cheng S., Song B., Zhong X., Lin X., Li W., . . Morse D. (2015) The Symbiodinium kawagutii genome illuminates dinoflagellate gene expression and coral symbiosis. Science 350, 691.

Little A. F., Oppen M. J., \& Willis B. L. (2004) Flexibility in algal symbioses shapes growth in reef corals. Science 304, 1492-1494. 
773 Littman R. A., van Oppen M. J. H., \& Willis B. L. (2008) Methods for sampling free-living

774

775

776

777

778

779

780

781

782

783

784

785

786

787

788

789

790

791

792

793

Symbiodinium (zooxanthellae) and their distribution and abundance at Lizard Island

(Great Barrier Reef). Journal of Experimental Marine Biology and Ecology 364, 48-53.

Logan D. D. K., LaFlamme A. C., Weis V. M., \& Davy S. K. (2010) FLOW-CYTOMETRIC

CHARACTERIZATION OF THE CELL-SURFACE GLYCANS OF SYMBIOTIC

DINOFLAGELLATES (SYMBIODINIUM SPP.)1. Journal of Phycology 46, 525-533.

Luo R., Liu B., Xie Y., Li Z., Huang W., Yuan J., . . Liu Y. (2015) SOAPdenovo2: an

empirically improved memory-efficient short-read de novo assembler. GigaScience 4, s13742-13015.

Luu K., Bazin E., \& Blum M. G. B. (2017) pcadapt: an R package to perform genome scans for selection based on principal component analysis. Molecular Ecology Resources 17, 6777.

Manning M. M., \& Gates R. D. (2008) Diversity in populations of free-living Symbiodinium from a Caribbean and Pacific reef. Limnology and Oceanography 53, 1853.

Martin M. (2011) Cutadapt removes adapter sequences from high-throughput sequencing reads. EMBnet. journal 17, 10-12.

McNeill D. F., Budd A. F., \& Borne P. F. (1997) Earlier (late Pliocene) first appearance of the Caribbean reef-building coral Acropora palmata: Stratigraphic and evolutionary implications. Geology 25, 891-894.

Miller M. W. (1995) Growth of a temperate coral: effects of temperature, light, depth, and heterotrophy. Marine Ecology Progress Series 122, 217-225. 
Mungpakdee S., Shinzato C., Takeuchi T., Kawashima T., Koyanagi R., Hisata K., . . Shoguchi Dinoflagellate Plastid Genome. Genome biology and evolution 6, 1408-1422.

797

798

799

800

801

802

803

804

805

806

807

808

809

810

811

812

813

814

815

816

Muscatine L., Goiran C., Land L., Jaubert J., Cuif J.-P., \& Allemand D. (2005) Stable isotopes ( $\delta \& 1 t ;$ sup\&gt;13\&lt;/sup\&gt;C and $\delta \& 1 t ; \sup \& g t ; 15 \& 1 t ; /$ sup\&gt;N) of organic matrix from coral skeleton. Proceedings of the National Academy of Sciences of the United States of America 102, 1525.

Muscatine L., Porter J. W., \& Kaplan I. R. (1989) Resource partitioning by reef corals as determined from stable isotope composition. Marine Biology 100, 185-193.

Narasimhan V., Danecek P., Scally A., Xue Y., Tyler-Smith C., \& Durbin R. (2016) BCFtools/RoH: a hidden Markov model approach for detecting autozygosity from nextgeneration sequencing data. Bioinformatics 32, 1749-1751.

O’Donnell K. E., Lohr K. E., Bartels E., Baums I. B., \& Patterson J. T. (2018) Acropora cervicornis genet performance and symbiont identity throughout the restoration process. Coral Reefs 37, 1109-1118.

Parkinson J. E., Banaszak A. T., Altman N. S., LaJeunesse T. C., \& Baums I. B. (2015a) Intraspecific diversity among partners drives functional variation in coral symbioses. Scientific reports 5, 15667.

Parkinson J. E., \& Baums I. B. (2014) The extended phenotypes of marine symbioses: ecological and evolutionary consequences of intraspecific genetic diversity in coralâ€"algal associations. Frontiers in microbiology 5, 445.

Parkinson J. E., Coffroth M. A., \& LaJeunesse T. C. (2015b) New species of Clade B Symbiodinium (Dinophyceae) from the greater Caribbean belong to different functional 
guilds: $S$. aenigmaticum sp. nov., S. antillogorgium sp. nov., S. endomadracis sp. nov., and S. pseudominutum sp. nov. Journal of Phycology 51, 850-858.

819

820

821

822

823

824

825

826

827

828

829

830

831

832

833

834

835

836

837

838

839

Parkinson J. E., Tivey T. R., Mandelare P. E., Adpressa D. A., Loesgen S., \& Weis V. M. (2018) Subtle Differences in Symbiont Cell Surface Glycan Profiles Do Not Explain SpeciesSpecific Colonization Rates in a Model Cnidarian-Algal Symbiosis. Frontiers in microbiology 9,842 .

Pettay D. T., Wham D. C., Smith R. T., Iglesias-Prieto R., \& LaJeunesse T. C. (2015) Microbial invasion of the Caribbean by an Indo-Pacific coral zooxanthella. Proceedings of the National Academy of Sciences of the United States of America 112, 7513-7518.

Pinzón J. H., Kamel B., Burge C. A., Harvell C. D., Medina M., Weil E., \& Mydlarz L. D. (2015) Whole transcriptome analysis reveals changes in expression of immune-related genes during and after bleaching in a reef-building coral. Royal Society Open Science 2.

Pochon X., Putnam H. M., Burki F., \& Gates R. D. (2012) Identifying and characterizing alternative molecular markers for the symbiotic and free-living dinoflagellate genus Symbiodinium. PloS one 7, e29816-e29816.

Poland D. M., \& Coffroth M. A. (2017) Trans-generational specificity within a cnidarian-algal symbiosis. Coral Reefs 36, 119-129.

Pollock F. J., McMinds R., Smith S., Bourne D. G., Willis B. L., Medina M., . . Zaneveld J. R. (2018) Coral-associated bacteria demonstrate phylosymbiosis and cophylogeny. Nature Communications 9, 4921.

Precht W. F., Vollmer S. V., Modys A. B., \& Kaufman L. (2019) Fossil <i>Acropora prolifera $</ \mathbf{i}>$ (Lamarck, 1816) reveals coral hybridization is not only a recent phenomenon. Proceedings of the Biological Society of Washington 132, 40-55. 
840 Pritchard J. K., Stephens M., \& Donnelly P. (2000) Inference of population structure using multilocus genotype data. Genetics 155, 945-959.

842 Quinlan A. R., \& Hall I. M. (2010) BEDTools: a flexible suite of utilities for comparing genomic features. Bioinformatics 26, 841-842.

844 Reich H. G., Rodriguez I. B., LaJeunesse T. C., \& Ho T.-Y. (2020) Endosymbiotic dinoflagellates pump iron: differences in iron and other trace metal needs among the

847 Serrano X., Baums I., O'Reilly K., Smith T., Jones R., Shearer T., . . B Baker A. (2014) Geographic differences in vertical connectivity in the Caribbean coral Montastraea cavernosa despite high levels of horizontal connectivity at shallow depths. Molecular ecology 23, 4226-4240.

Serrano X., Baums I. B., Smith T. B., Jones R. J., Shearer T. L., \& Baker A. C. (2016) Long distance dispersal and vertical gene flow in the Caribbean brooding coral Porites astreoides. Scientific reports $\mathbf{6}$.

Shah S., Chen Y., Bhattacharya D., \& Chan C. X. (2020) Sex in Symbiodiniaceae dinoflagellates: genomic evidence for independent loss of the canonical synaptonemal complex. Scientific reports $\mathbf{1 0}, 9792$.

Shinzato C., Shoguchi E., Kawashima T., Hamada M., Hisata K., Tanaka M., . . Satoh N. (2011) Using the Acropora digitifera genome to understand coral responses to environmental change. Nature 476, 320-323.

Shoguchi E., Beedessee G., Tada I., Hisata K., Kawashima T., Takeuchi T., .. . Shinzato C. (2018) Two divergent Symbiodinium genomes reveal conservation of a gene cluster for sunscreen biosynthesis and recently lost genes. BMC genomics 19, 458 . 
863 Shoguchi E., Shinzato C., Kawashima T., Gyoja F., Mungpakdee S., Koyanagi R., . . Fujiwara

864

865

866

867

868

869

870

871

872

873

874

875

876

877

878

879

880

881

882 Tamura K., Stecher G., Peterson D., Filipski A., \& Kumar S. (2013) MEGA6: molecular

883

884

M. (2013) Draft assembly of the Symbiodinium minutum nuclear genome reveals dinoflagellate gene structure. Current Biology 23, 1399-1408.

Silverstein R. N., Cunning R., \& Baker A. C. (2017) Tenacious D: Symbiodinium in clade D remain in reef corals at both high and low temperature extremes despite impairment. The Journal of Experimental Biology.

Simão F. A., Waterhouse R. M., Ioannidis P., Kriventseva E. V., \& Zdobnov E. M. (2015) BUSCO: assessing genome assembly and annotation completeness with single-copy orthologs. Bioinformatics 31, 3210-3212.

Sogin E. M., Anderson P., Williams P., Chen C.-S., \& Gates R. D. (2014) Application of 1HNMR Metabolomic Profiling for Reef-Building Corals. PloS one 9, e111274.

Stamatakis A. (2014) RAxML version 8: a tool for phylogenetic analysis and post-analysis of large phylogenies. Bioinformatics 30, 1312-1313.

Stanke M., Keller O., Gunduz I., Hayes A., Waack S., \& Morgenstern B. (2006) AUGUSTUS: ab initio prediction of alternative transcripts. Nucleic acids research 34, W435-W439.

Stanke M., Steinkamp R., Waack S., \& Morgenstern B. (2004) AUGUSTUS: a web server for gene finding in eukaryotes. Nucleic acids research 32, W309-W312.

Stanley G. D. (2006) Photosymbiosis and the Evolution of Modern Coral Reefs. Science 312, 857. evolutionary genetics analysis version 6.0. Molecular Biology and Evolution 30, 27252729. 
885 Terraneo T. I., Fusi M., Hume B. C. C., Arrigoni R., Voolstra C. R., Benzoni F., . . Berumen M.

886

887

888

889

890

891

892

893

894

895

896

897

898

899

900

901

902

903

904

905

906

907

L. (2019) Environmental latitudinal gradients and host-specificity shape Symbiodiniaceae distribution in Red Sea Porites corals. Journal of Biogeography 46, 2323-2335.

Thornhill D. J., Fitt W. K., \& Schmidt G. W. (2006a) Highly stable symbioses among western Atlantic brooding corals. Coral Reefs 25, 515-519.

Thornhill D. J., Howells E. J., Wham D. C., Steury T. D., \& Santos S. R. (2017) Population genetics of reef coral endosymbionts (Symbiodinium, Dinophyceae). Molecular ecology 26, 2640-2659.

Thornhill D. J., LaJeunesse T. C., Kemp D. W., Fitt W. K., \& Schmidt G. W. (2006b) Multiyear, seasonal genotypic surveys of coral-algal symbioses reveal prevalent stability or post-bleaching reversion. Marine Biology 148, 711-722.

Thrall P. H., Hochberg M. E., Burdon J. J., \& Bever J. D. (2007) Coevolution of symbiotic mutualists and parasites in a community context. Trends in Ecology \& Evolution 22, 120126.

Trench R. K. (1979) The cell biology of plant-animal symbiosis. Annual Review of Plant Physiology 30, 485-531.

UniProt C. (2014) UniProt: a hub for protein information. Nucleic acids research 43, D204D212.

Van Oppen M. J. H., Willis B. L., Vugt H. V., \& Miller D. J. (2000) Examination of species boundaries in the Acropora cervicornis group (Scleractinia, Cnidaria) using nuclear DNA sequence analyses. Molecular ecology 9, 1363-1373.

Vollmer S. V., \& Palumbi S. R. (2002) Hybridization and the Evolution of Reef Coral Diversity. Science 296, 2023. 
908

909

910

911

912

913

914

915

916

917

918

919

920

921

922

923

924

925

926

927

928

929

930

Vollmer S. V., \& Palumbi S. R. (2006) Restricted Gene Flow in the Caribbean Staghorn Coral Acropora cervicornis: Implications for the Recovery of Endangered Reefs. Journal of Heredity 98, 40-50.

Warren R. L., Yang C., Vandervalk B. P., Behsaz B., Lagman A., Jones S. J. M., \& Birol I. (2015) LINKS: Scalable, alignment-free scaffolding of draft genomes with long reads. GigaScience 4, 35.

Weber L., González-Díaz P., Armenteros M., \& Apprill A. (2019) The coral ecosphere: A unique coral reef habitat that fosters coral-microbial interactions. Limnology and Oceanography 9999, 1-16.

Weis V. M., Reynolds W. S., deBoer M. D., \& Krupp D. A. (2001) Host-symbiont specificity during onset of symbiosis between the dinoflagellates Symbiodinium spp. and planula larvae of the scleractinian coral Fungia scutaria. Coral Reefs 20, 301-308.

Williams G. J., Sandin S. A., Zgliczynski B. J., Fox M. D., Gove J. M., Rogers J. S., . . Smith J. E. (2018) Biophysical drivers of coral trophic depth zonation. Marine Biology 165, 60.

Wood-Charlson E. M., Hollingsworth L. L., Krupp D. A., \& Weis V. M. (2006) Lectin/glycan interactions play a role in recognition in a coral/dinoflagellate symbiosis. Cellular Microbiology 8, 1985-1993.

Xu H., Luo X., Qian J., Pang X., Song J., Qian G., . . Chen S. (2012) FastUniq: a fast de novo duplicates removal tool for paired short reads. PloS one 7, e52249.

Zhang W., Xu J., Liu D., Liu H., Lu X., \& Yu W. (2018) Characterization of an $\alpha$-agarase from Thalassomonas sp. LD5 and its hydrolysate. Applied Microbiology and Biotechnology 102, 2203-2212. 


\section{Data availability}

932 Sequences are under NCBI SRA PRJNA473816. Code for data analysis and figure generation is

933 available on github (https://github.com/hgreich/Sfitti).

934

\section{Author contributions}

936 Conceived the project: SAK, IBB, HGR. Obtained funding: IBB, NDF. Mentorship: IBB, SAK.

937 Field collections of corals: SAK, IBB, NDF. Molecular work: SAK, MDD. Bioinformatic

938 analyses: HGR, SAK, KHS. Wrote the paper: HGR, SAK, IBB, NDF, KHS.

939

940 Tables

941

942 Table S1: Sample information including host taxa, location, sampling depth, sequencing

943 platform, and accession number. Per sample information on read counts, mapping rates, and SNP

944 summary statistics are also included. Multi-locus genotypes from 12 microsatellite loci, 58,538

945 "high quality" SNPs, and 6,813 "conservative" SNPs with no missing data are included.

946

947 Table S2: Genome assembly summary statistics for Symbiodinium 'fitti' sample PFL14120.

949 Table S3: Annotation information for the 24,000+ genes in Symbiodinium 'fitti.'

951 Table S4: Annotation information and selection outlier statistics for Symbiodinium 'fitti' SNPS

$952(12,700)$ that are predicted to fall in coding regions. 
954 Table S5: AMOVA of indicates that $\sim 12 \%$ of Symbiodinium 'fitti' variation is due to host taxon

955 whereas negative \% variation is due to location.

956

957 Table S6: Summary statistics from Structure Harvester including the Evanno’s delta K method

958 which predicted three main clusters.

959

960 Table S7: Selection outlier statistics and per SNP summary statistics loci identified as selection

961 outliers by BayeScan (370) and PCAdapt (4,987). 307 selection outlier SNPs were shared

962 between the two programs.

963

964 Table S8: Summary statistics for the predicted downstream effects of SNPs (generated by the

965 SNPeff program). 60,373 (84.43\%) modifier/non-coding, 3,629 (5.08\%) moderate/mostly

966 harmless, 7,451 (10.42\%) low impact variants that might change protein efficiency/effectiveness,

967 and $51(0.07 \%)$ highly disruptive SNPs were identified.

968

969 Table S9: Predicted downstream effects (SnpEff) and annotation information for variants in

970 coding regions.

971

972 Table S10: Predicted downstream effects (SnpEff) and annotation information for 12 variants in

973 coding regions that were identified as selection outliers by both PCAdapt and BayeScan.

974

\section{$975 \quad$ Figures}

976 
Distribution of Symbiodinium 'fitti' samples
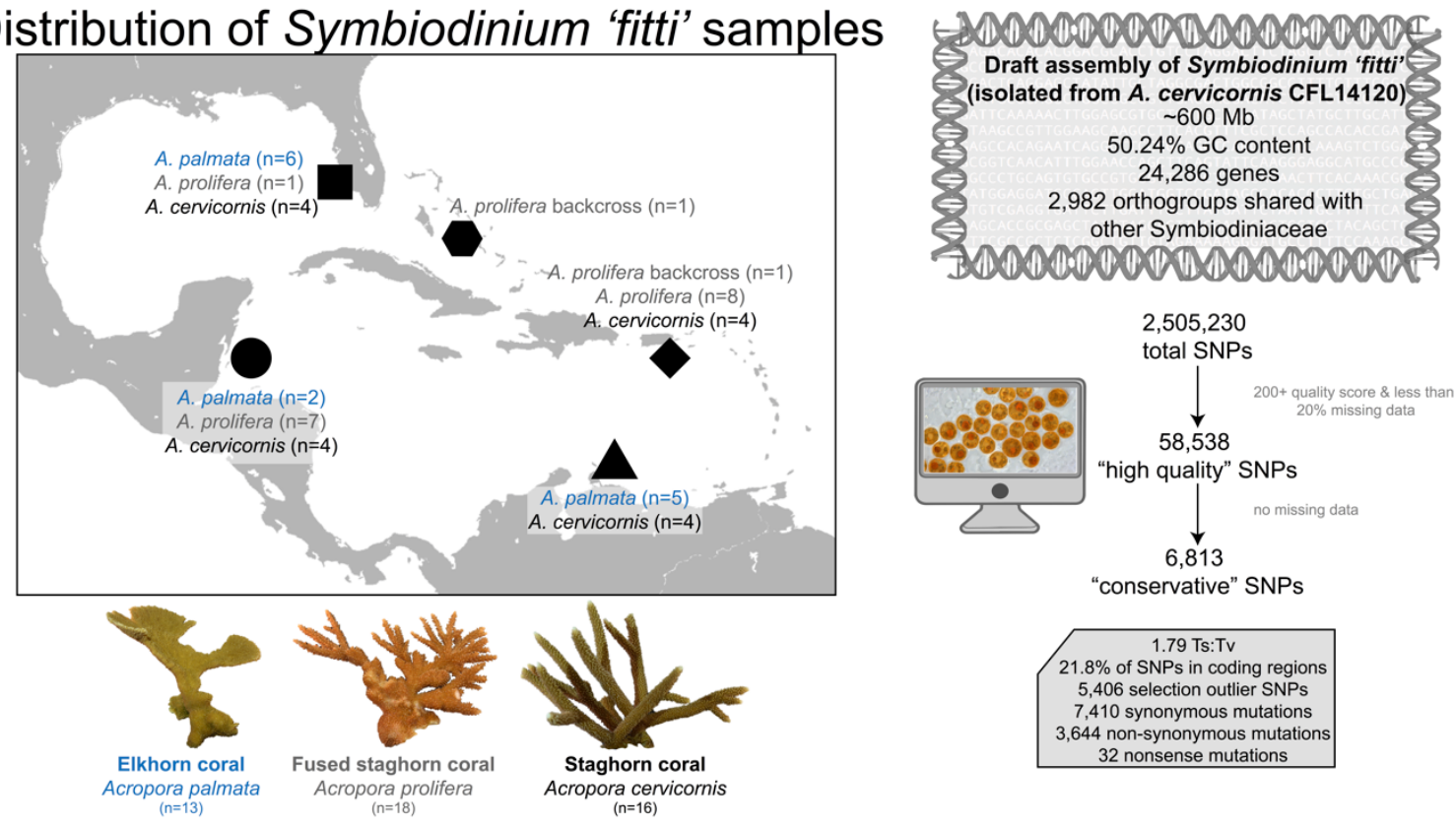

"conservative" SNPs

978 Fig. 1: Sampling design and summary statistics of population genomic approach used to

979 characterize Symbiodinium 'fitti' across three coral hosts. Acropora palmata $(\mathrm{n}=13)$, Acropora

980 cervicornis $(\mathrm{n}=16)$, and Acropora prolifera $(\mathrm{n}=18)$ samples used for shallow genome

981 sequencing spanned their geographic distribution. Summary statistics for the 'deeply-sequenced'

982 CFL 14120 A. cervicornis - S. 'fitti' draft genome assembly including overall length, \%GC

983 content, \# of genes, and shared gene families with other Symbiodiniaceae. Summary statistics

984 and visual depiction of quality filtering work flow that was employed to identify high quality

985 variants and those that are under selection. Coral images from N. Fogarty and I. Baums. 


\section{$\mathbf{5 8 , 5 3 8}$ 'genotyping' Symbiodinium 'fitti' SNPs}

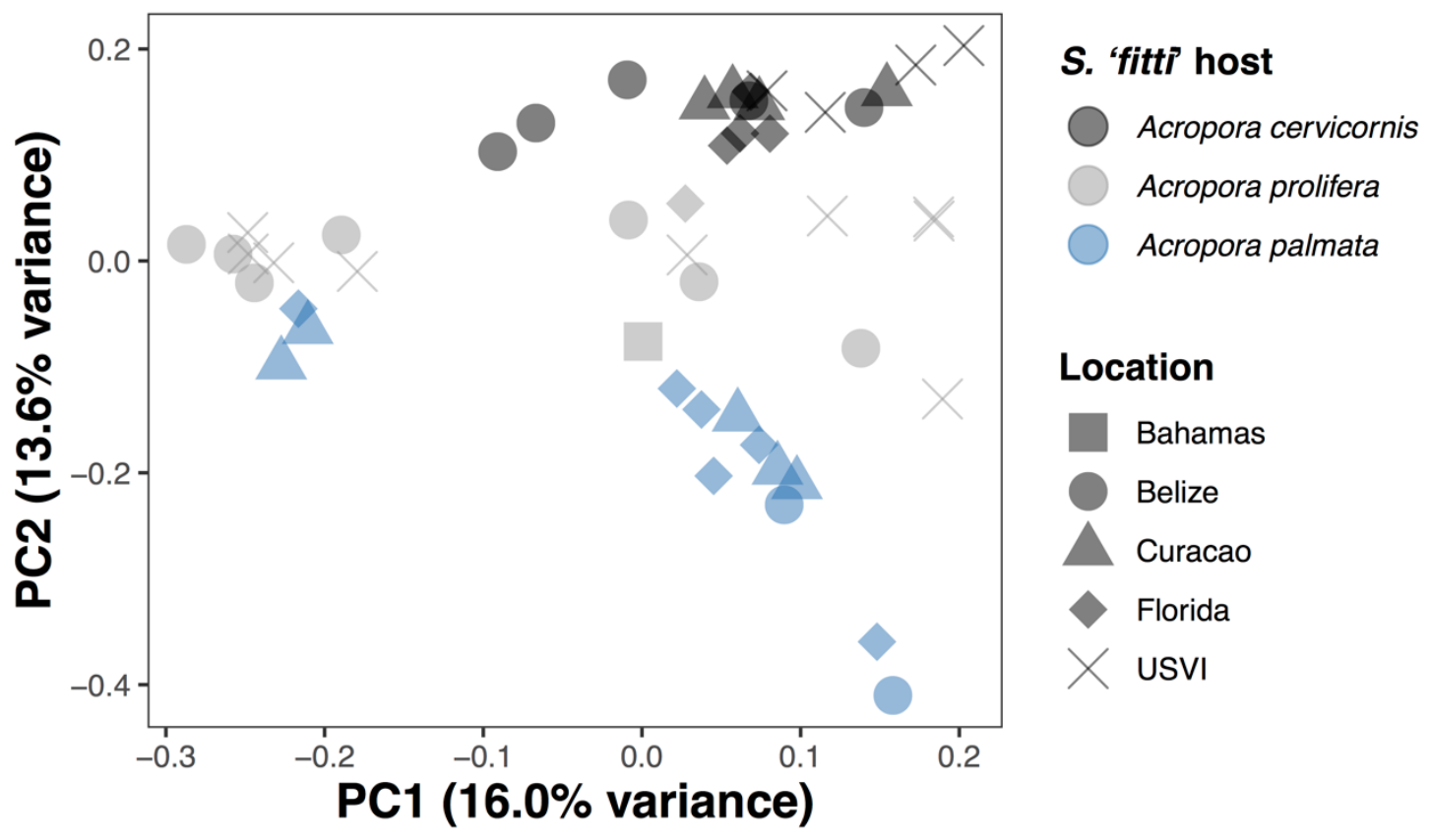

991

992 Fig. 2: Principal component analysis (PCA) of 58,583 genotyping Symbiodinium 'fitti' SNPs

993 illustrates genomic differentiation by host taxon. Coral images from N. Fogarty and I. Baums. 


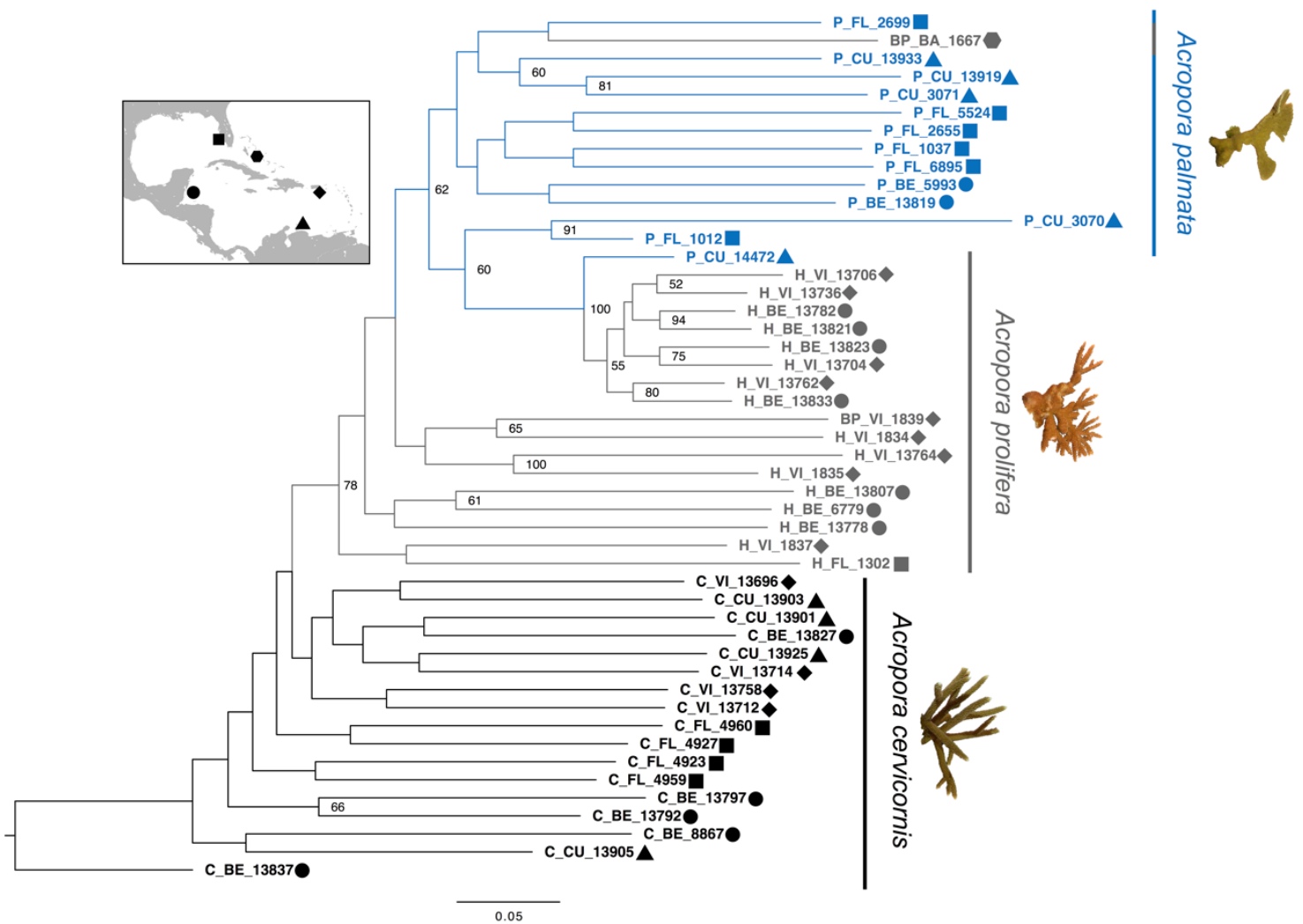

996 Fig. 3: Allelic composition of Symbiodinium 'fitti' is at the sub-species level. RAxML

997 (Maximum Likelihood) phylogeny of 6,813 “conservative” genotyping S. 'fitti' SNPs without

998 missing data and 100 bootstrap replicates illustrate $S$. 'fitti' genomic differentiation by host

999 taxon.

1000

1001

1002

1003

1004

1005

1006

1007 


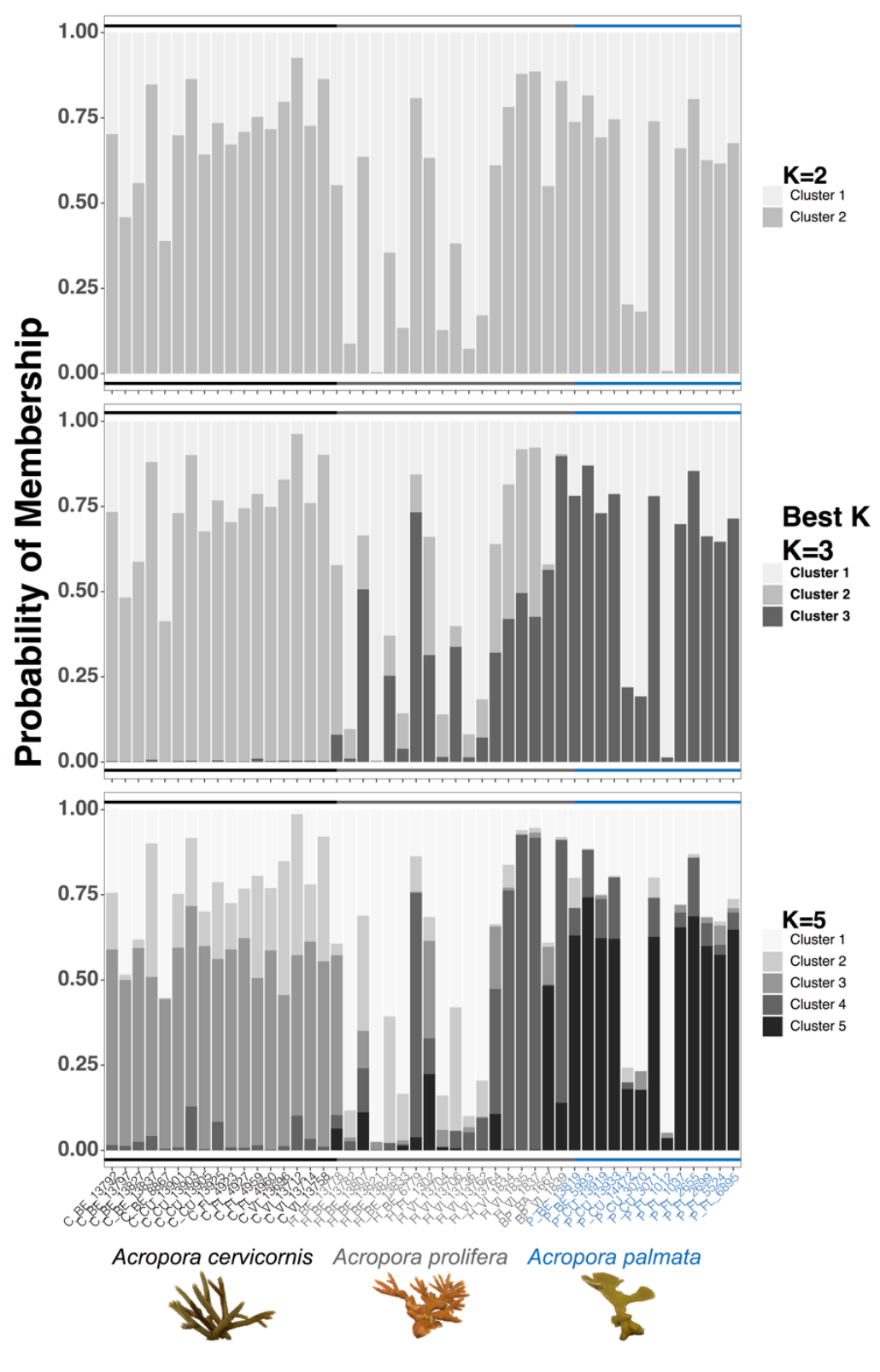

1009 Fig. 4: Symbiodinium 'fitti' strain population assignment aligns with host taxon. Probability of 1010 membership predicted by STRUCTURE for 58,583 "high quality” Symbiodinium 'fitti' SNPS.

$1011 \mathrm{~K}=3$ was determined as best $\mathrm{K} . \mathrm{K}=2$ and $\mathrm{K}=5$ are also presented for comparison. These results 1012 illustrate that $S$. 'fitti' membership clusters largely correspond to their host acroporid taxon. 1013 Coral images from N. Fogarty and I. Baums. 


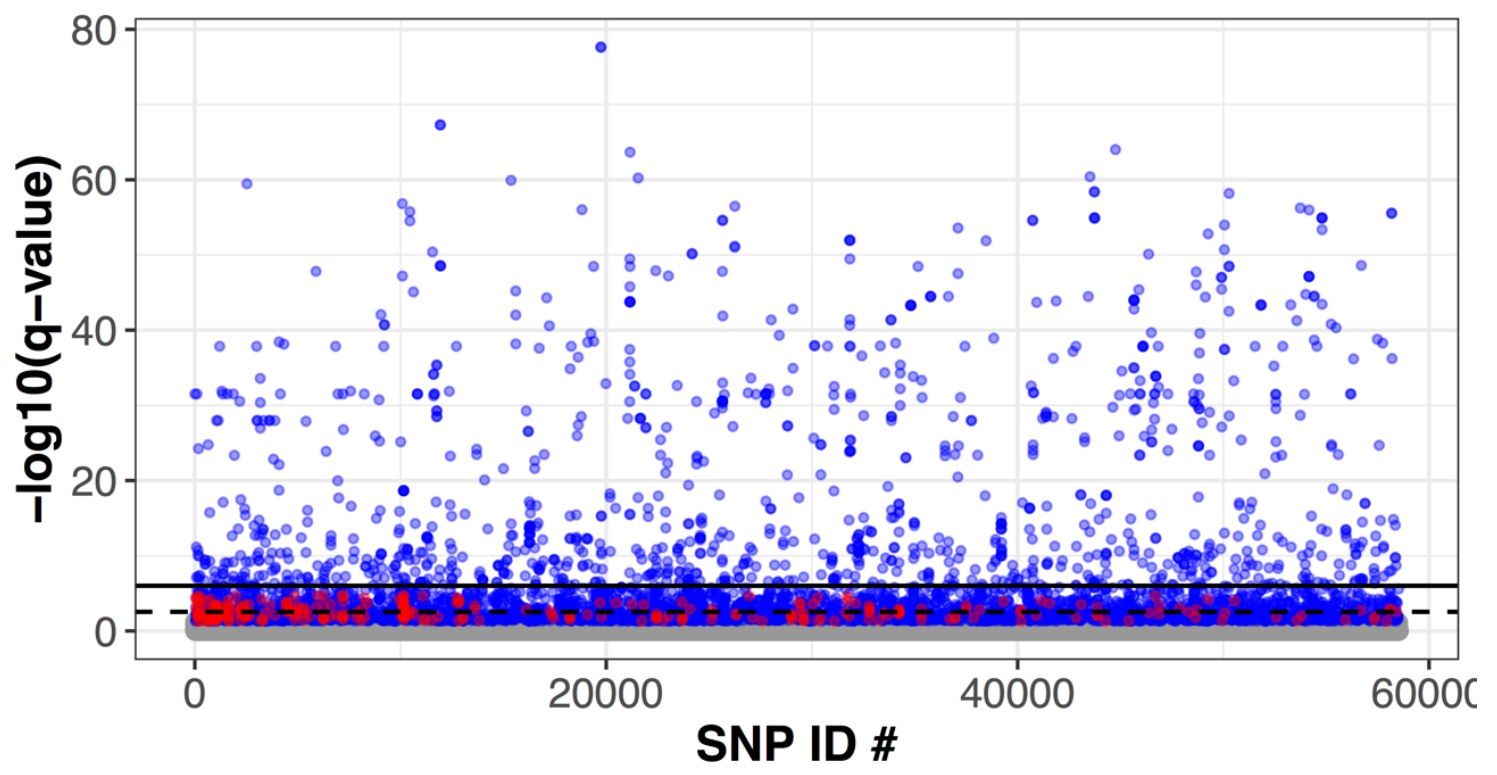

Outlier Type $\bullet$ Not Significant $\bullet$ PCAdapt $\bullet$ BayeScan

1016 Fig. 5: Genetic variants of $S$. 'fitti' showing signatures of selection. Manhattan plot of $-\log 10$ transformed q-values for 58,583 genotyping SNPs. SNPs are highlighted by outlier detection

1018 program (PCAdapt loci highlighted in blue; BayeScan loci highlighted in red). 339 selection

1019 outlier SNPs were shared between the two programs. 103 outlier loci identified by BayeScan had

1020 a Bayes probability of 1 and q-value of 0 which becomes infinite following logarithmic

1021 transformation and were therefore removed from the plot. These loci were also had high

1022 BayeScan fixation levels between host and location. The dashed line represents the 5\% FDR

1023 adjustment threshold (2.54) and whereas the solid line represents the 0.05 Bonferroni correction

1024 threshold (6.01).

1025

1026

1027

1028 


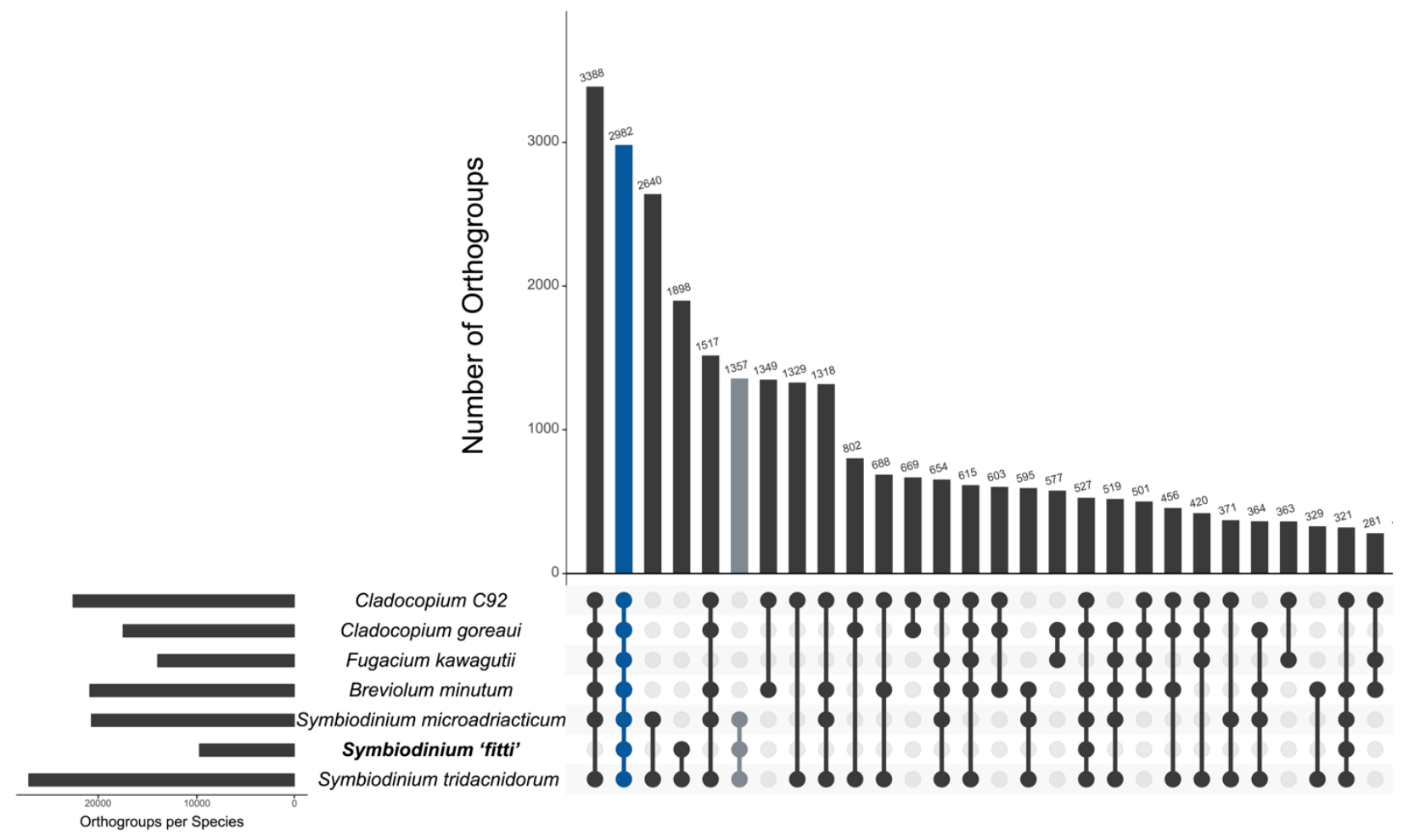

Fig. S1: Gene families shared between different Symbiodiniaceae lineages (including 


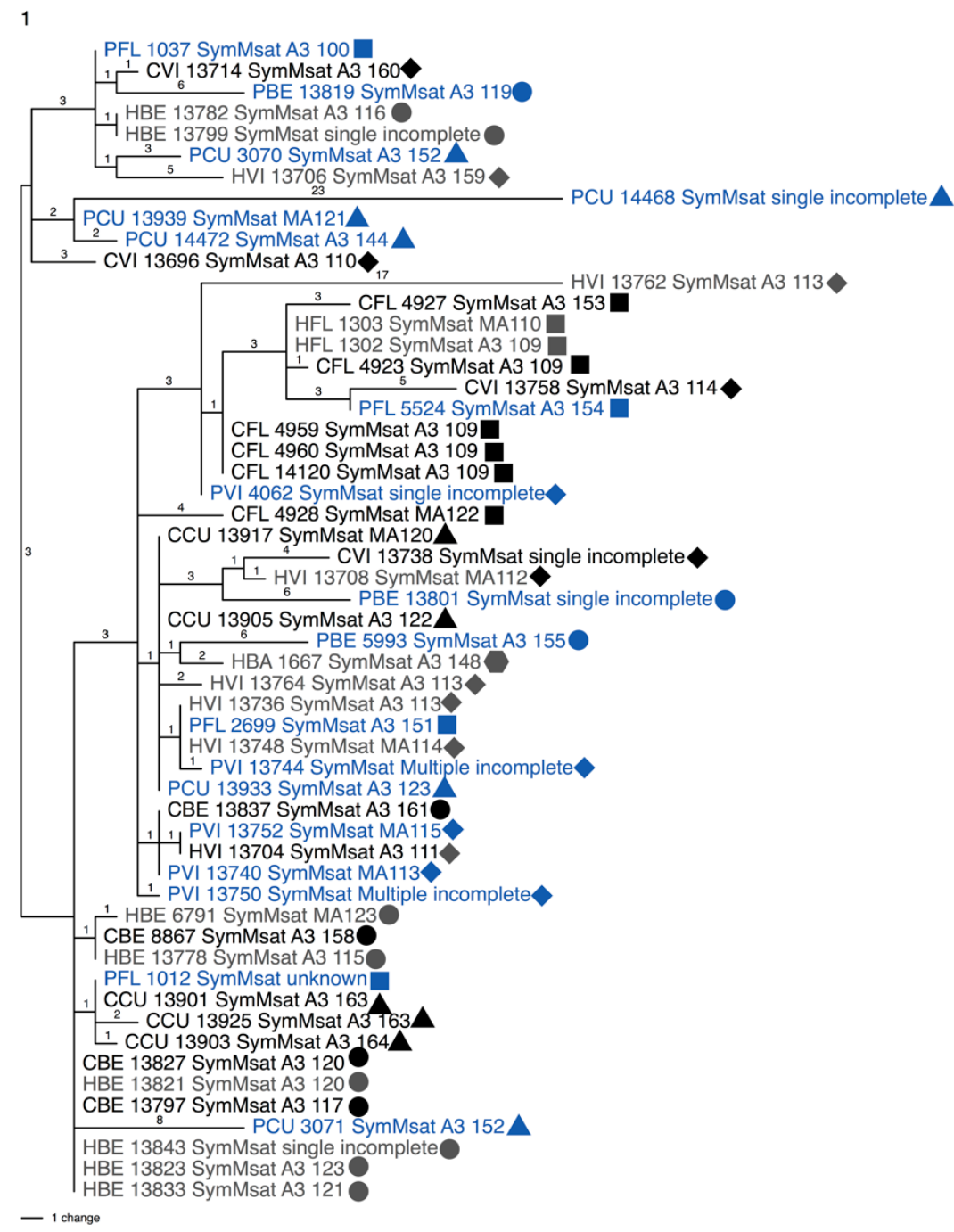

1044 Fig. S2: Intragenomic variation of Symbiodinium 'fitti' is at the sub-species level. Phylogeny of

1045 the $p s b A$ non-coding region, a commonly used marker used to help delimit Symbiodinium species

1046 indicates that $S$. 'fitti' in its three different hosts are all one species. 

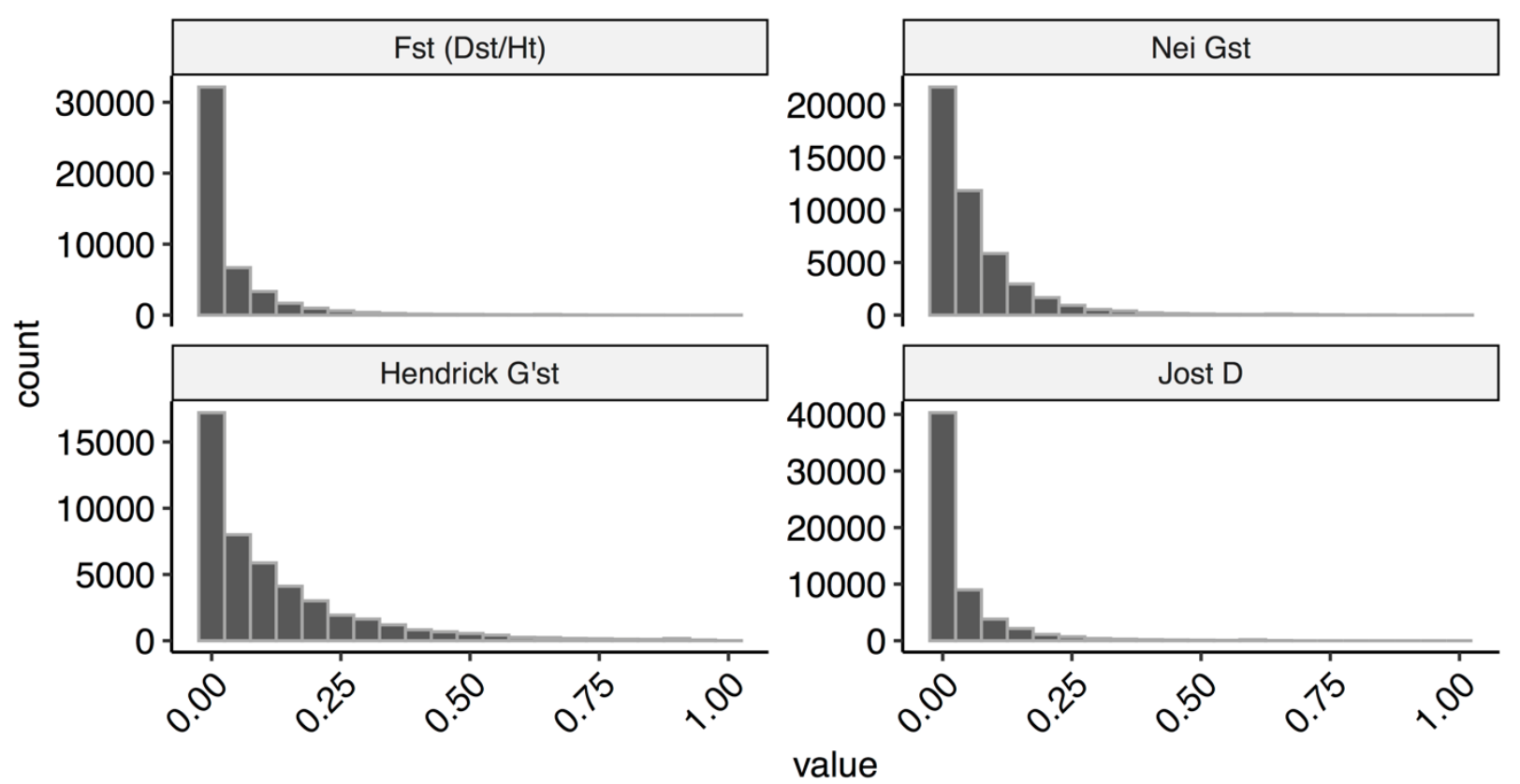

1052 Fig. S3: Histogram of per SNP fixation levels for 58,538 genotyping SNPs. 